\title{
Dynamics of an impact oscillator near a degenerate graze
}

\author{
D R J Chillingworth \\ School of Mathematics, University of Southampton, Southampton SO17 1BJ, UK \\ E-mail: drjc@maths.soton.ac.uk
}

\begin{abstract}
We give a complete analysis of low-velocity dynamics close to grazing for a generic one degree of freedom impact oscillator. This includes nondegenerate (quadratic) grazing and minimally degenerate (cubic) grazing, corresponding respectively to nondegenerate and degenerate chatter. We also describe the dynamics associated with generic one-parameter bifurcation at a more degenerate (quartic) graze, showing in particular how this gives rise to the often-observed highly convoluted structure in the stable manifolds of chattering orbits. The approach adopted is geometric, using methods from singularity theory.
\end{abstract}

AMS classification scheme numbers: 34A36,34C23,37B55,70K40,70K50,70G60

Submitted to: Nonlinearity

\section{Introduction}

The theory of forced nonlinear oscillations has well known and widespread applications in science and engineering: see Hayashi [24], Fidlin [20], for example, as well as landmark texts Andronov et al. [2], Schmidt et al. [37], Guckenheimer et al. [23]. However, there are many engineering contexts in which oscillations are a highly undesirable feature of performance, especially when these lead to impacts between moving parts or against rigid supports, generating noise and wear and ultimately leading to mechanical breakdown: for some examples see Alzate et al. [1], Mason et al. [31], Stewart [40], Pavlovskaia et al. [35] as well as those described in books such as Babitsky et al. [5], Brogliato [8] and di Bernardo et al. [7].

A simple model for such dynamical impact phenomena (often called vibro-impact systems) is the one degree of freedom impact oscillator. This consists of a forced nonlinear oscillator

$$
\ddot{x}+f(x, \dot{x})=g(t)
$$


for $x \in \mathbf{R}$, together with a constraint $x \geq c$ where $c$ is a constant often called the clearance. Here $f$ and $g$ are smooth $\ddagger$ functions, and as usual the dot denotes $d / d t$.

The model must include a prescription for how the dynamics are to proceed when $x=c$. This will typically be given by a restitution rule that whenever $(x, \dot{x})=(c, v)$ then the velocity $v$ is instantaneously replaced by $\tilde{v}$ (a function of $v$ and perhaps other variables) satisfying $\tilde{v} v<0$. The simplest practical example is a linear rule with $\tilde{v}=-r v$ where $0 \leq r \leq 1$, which includes the cases of perfect adherence $(r=0)$ and perfectly elastic restitution $(r=1)$. There are many variations on this model, involving more than one degree of freedom and/or more than one constraint or a non-instantaneous restitution rule. In this paper we shall require only that the linearisation of $\tilde{v}$ at $v=0$ takes the form $-r v$ with $0<r<1$; thus we exclude the extreme cases.

The fact that a simple impact system can give rise to extremely complicated dynamics has long been noted, and there are many analytical and numerical studies showing complicated periodicities or convoluted orbit structures in phase space: see for example Thompson et al. [44], Shaw et al. [38], Hindmarsh et al. [25], Peterka et al. [36], Kleczka et al. [30], Virgin et al. [46], Wagg et al. [47],[48], Valente et al. [45]. Nevertheless, even a 1-degree of freedom linear oscillator with a linear restitution rule is still poorly understood from the overall dynamical point of view.

A more systematic attack on the global problem was undertaken by Whiston in a pioneering series of papers [49],[50],[51]; this enterprise inspired much subsequent work including Budd et al. [15], Lamba [28], Nordmark [33] as well as the present paper.

A key feature of impact oscillator dynamics that gives it a different flavour from (smooth) nonlinear dynamics is the phenomenon of grazing where a periodic orbit reaches the obstacle with zero velocity and nonzero acceleration: small perturbations of such orbits may or may not exhibit impacts. The resulting bifurcation phenomena have been studied by many authors including Budd et al. [14], Chin et al. [17], Dankowitz et al. [18], Foale et al. [21], Ivanov [29], Nordmark [33],[34], Szalai et al. [42], Molenaar et al. [32], Zhao et al. [53].

Another important aspect of impact oscillator dynamics that attracts particular interest is the low-velocity behaviour close to impact, characterised by the phenomenon of chatter. On leaving $x=c$ with small positive velocity but negative acceleration the orbit (i.e. the solution to (1)) may return to hit $x=c$ again after a short time; after restitution at the impact the process will repeat, and under suitable conditions the orbit may come to rest at $x=c$ after a finite time but an infinite number of such impacts. It will remain stuck at the obstacle $x=c$ until such time as the acceleration (from the forcing) becomes positive and it once again lifts off into the region $x>c$. See for example Demeio et al. [19] for a simple mechanical realisation of this phenomenon, or Stone et al. [41], Valente et al. [45] for more complicated dynamical settings. The first systematic

$\ddagger$ For simplicity we take smooth to mean $C^{\infty}$, although many of the results will clearly hold with less differentiability. 
analytic and geometrical analysis of the dynamics of chatter was carried out by Budd et al. [14], using a combination of insightful numerics supported theoretically by first and second order approximations. In this paper we extend those results by providing a complete and rigorous analysis of chatter for generic impact oscillator systems (1) and for generic 1-parameter families of such systems.

In many applications the function $g$ in (1) is periodic with period $T>0$. In this case a standard technique for studying a forced oscillator such as (1) without the impacts is to focus on the the discrete dynamical system $F$ that samples the solutions at times $\{n T: n \in \mathbf{Z}\}$ : then periodic solutions of (1) correspond to periodic orbits of $F$, and so on. When impacts are introduced, however, this is no longer appropriate since the influence of the obstacle on a given trajectory cannot be expected to be $T$ periodic. A common approach to dealing with this problem, used by Shaw et al. [38], Thompson et al. [44], Whiston [50] and others, is first to regard (1) as an autonomous system in $\mathbf{R}^{3}$ by including the equation $\dot{t}=1$ and then to consider the plane $x=c$ and the discrete dynamical system defined on it by the first return of trajectories after impacting the obstacle. This fits naturally with the geometry of the physical description of (1), but the resulting discrete system now has loci of discontinuity that arise from grazing trajectories. It is not easy in this setting to see how the discontinuities and the associated dynamical phenomena respond to change in initial conditions and/or governing parameters such as the clearance.

In this paper we use an alternative geometric approach, first set out in [16], which allows greater insight into the geometry of discontinuities and their influence on dynamics by replacing the picture of curved orbits intersecting a flat obstacle with an equivalent picture of straightened-out orbits intersecting a curved obstacle: the impact surface. See Section 2 below for the precise description. From this point of view, the problem of analysing the geometry of orbits at and near grazing becomes more readily amenable to the tools of local differential topology, dynamical systems and singularity theory.

Taking this approach, we look again at the phenomena of grazing and chatter in the light of the geometry of the impact surface. We first use this to provide rigorous underpinning for the key results in [14] that are based on approximations: by placing the results in a wider context we are able to give a full analysis of the local dynamical phenomena. We then extend this to cover the case of the discontinuous bifurcations that arise in the neighbourhood of a doubly-degenerate graze as the clearance or some other parameter is varied and the degeneracy is unfolded. In particular we exhibit the key mechanism for the creation of multiple-loop structures in stable manifolds of chatter points, which is a first step in understanding the multiple-loop patterns observed numerically for attractors and basins of attraction of periodic orbits in impact oscillators: see [50, Figure 17], [14, Figures 4 and 19], as well as [6, Figure 3] and [7] (in particular the front cover). Taken together, the results of the present paper provide a complete description of generic low-velocity impact dynamics and 1-parameter bifurcations. 


\section{Geometrical model for dynamics: the impact surface}

We begin by recalling the construction described in Chillingworth [16] for modelling the overall dynamics of a one degree of freedom impact oscillator. The model is based on a 2-dimensional surface in three dimensions that characterises the impacts, together with certain natural maps associated with it that represent the dynamics. A similar geometrical construction was used by Sotomayor et al. [39], albeit with a different dynamical interpretation, to classify local vector fields on a manifold with boundary.

\subsection{The impact surface and associated maps}

Let $x(b, v, \tau ; t)$ denote the unique solution to (1) with initial data

$$
(x, \dot{x})=(b, v) \quad \text { when } \quad t=\tau .
$$

For fixed choice of $c \in \mathbf{R}$ we then write

$$
x_{c}(\tau, v ; t):=x(c, v, \tau ; \tau+t)
$$

so that

$$
x_{c}(\tau, v ; 0)=c, \quad \dot{x}_{c}(\tau, v ; 0)=v .
$$

Definition 2.1 The impact surface $V_{c}$ is the locus of points among all the solutions where impact occurs, that is

$$
V_{c}:=\left\{(\tau, v ; t) \in \mathbf{R}^{3}: x_{c}(\tau, v ; t)=c\right\} .
$$

Note that in [16] the convention was to write $(v, \tau ; t)$ rather than $(\tau, v ; t)$ : we trust no confusion will arise from this notation reversal.

By definition $V_{c}$ contains the plane $t=0$, which we shall denote by $\Pi$ and which plays a key role in our formulation of the dynamics. It is proved in [16, Lemma 1] that $V_{c}$ is indeed a surface (smooth 2-manifold), with the exception of the points on the $\tau$-axis $\Pi_{0}$ and possibly certain other points where $v=0$, characterised precisely in terms of the functions $f$ and $g$ in (1) and the clearance $c$. It is shown§ in [16, Proposition 1] (see also [14] where it is inherent in the analysis, and see the calculations below) that in a neighbourhood of $\Pi_{0}$ the impact surface $V_{c}$ consists of two sheets (smooth 2-manifolds) intersecting transversely along $\Pi_{0}$. One of these is of course the plane $\Pi$ while the other sheet $V_{c}^{\prime}$ is represented in a neighbourhood of $\Pi_{0}$ as the graph of a smooth function $v=v_{c}(\tau, t)$ given implicitly by

$$
y_{c}\left(\tau, v_{c} ; t\right)=0
$$

when $x_{c}$ is written in the form

$$
x_{c}=c+t y_{c}(\tau, v ; t)
$$

$\S$ The statement $r \geq 2$ there is an error: it originally referred to degree of differentiability, and has no connection with the restitution coefficient here. 
for a smooth function $y_{c}$ on a neighbourhood of $\Pi_{0}$ in $\mathbf{R}^{3}$.

By implicit differentiation of (3) we find

$$
\left.\frac{\partial}{\partial t} v_{c}(\tau, t)\right|_{t=0}=-\frac{1}{2} a_{c}(\tau)
$$

where $a_{c}(\tau):=\ddot{x}_{c}(\tau, 0 ; 0)$ is the initial acceleration (again see [14] as well as [16, Proposition 1]).

There are three maps which we use to build the dynamical model for the impact oscillator. We now describe each of these in turn.

\subsubsection{The projection $p_{c}$. Consider the projection}

$$
p: \mathbf{R}^{3} \rightarrow \Pi:(\tau, v ; t) \mapsto(\tau, v)
$$

from $\mathbf{R}^{3}$ to the plane $\Pi$ of initial data, and let $p_{c}$ denote its restriction to $V_{c}^{\prime}$ :

$$
p_{c}:=p \mid V_{c}^{\prime}: V_{c}^{\prime} \rightarrow \Pi
$$

Let $H_{c}$ be the set of singular points of $p_{c}: V_{c}^{\prime} \rightarrow \Pi$ (including those, if any, where $V_{c}^{\prime}$ itself may be singular). From the Implicit Function Theorem it is immediate that

$$
H_{c}=\left\{(\tau, v ; t) \in V_{c}^{\prime}: \dot{x}_{c}(\tau, v ; t)=0\right\}
$$

which in geometric terms is the horizon of $V_{c}^{\prime}$ as viewed along the $t$-direction. Dynamically, the points of $H_{c}$ represent graze points, that is points where the solution to (1) meets the obstacle $x=c$ with zero velocity. See Figure 1, where for diagrammatic simplicity the curve $H_{c}$ is not distinguished from the horizon of $V_{c}^{\prime}$ as seen from the viewpoint of the reader: in reality these are of course different. The subset $P_{c}:=p\left(H_{c}\right)$ of $\Pi$ is the apparent outline (classically the apparent contour) of the surface $V_{c}^{\prime}$ in the t-direction: see Bruce [10], Bruce et al. [12].

The generic local geometry of smooth maps from a smooth surface $M$ into $\mathbf{R}^{2}$ is well understood since the work of Whitney [52]. The set of singular points is a smooth 1-manifold in $M$ consisting of arcs of fold points (where the singular set maps locally to a smooth 1-manifold) and isolated cusp points (where the singular set maps locally to a $\frac{3}{2}$-power cusp). The precise characterisations are given in terms of partial derivatives, and local coordinates can be chosen so that at fold points or cusp points the map has the form $(x, y) \mapsto\left(x, y^{2}\right)$ or $(x, y) \mapsto\left(x, x y+y^{3}\right)$ respectively. These generic results apply also to the conceivably more restricted setting of projection of a surface in $\mathbf{R}^{3}$ to a plane, and thus to the geometry of apparent outlines. In Figure 1 the points $w_{1}, w_{3}$ are images of fold points, while $w_{2}$ is the image of a cusp point.

As the surface or the view direction varies with one or more parameters the outline will typically undergo certain local transitions. The generic 1-parameter transitions in apparent outlines are also well understood: see [3],[9],[13] and Figure 2.

In general, without any restriction on $t$, there may be regions of $\Pi$ filled densely by arcs of $P_{c}$. However, if $V_{c}$ is periodic in $t$ (as may happen for undamped linear systems 


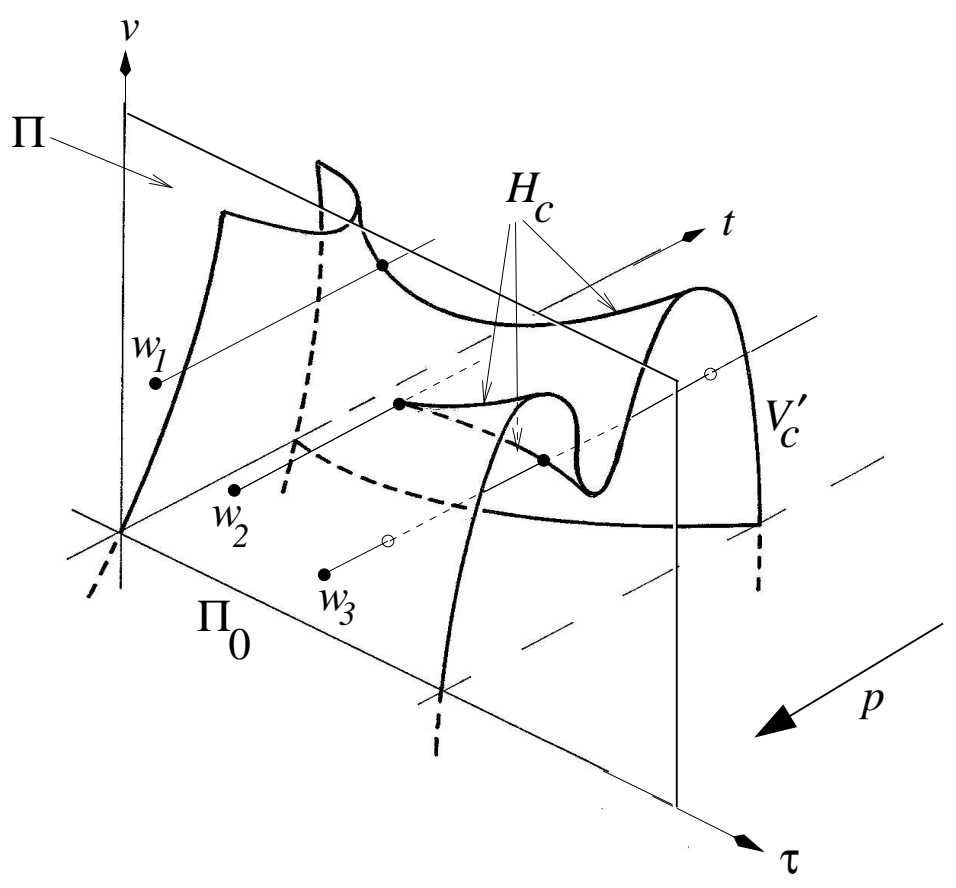

Figure 1. The impact surface $V_{c}=\Pi \cup V_{c}^{\prime}$ with horizon $H_{c}$ that is the set of singular points of the projection $p \mid V_{c}^{\prime} \rightarrow \Pi$. Points $w_{1}, w_{2}, w_{3}$ belong to the apparent outline $P_{c}=p\left(H_{c}\right)$ (not shown).

with periodic forcing) or we restrict to a compact subset of $V_{c}$ then this complication will not arise.

It is pertinent to ask whether for arbitrary choice of clearance $c$ the apparent outline of the impact surface $V_{c}^{\prime}$, which is determined by solutions of the differential equation (1), is indeed generic as just described, and whether the transitions in the apparent outline as $c$ varies are also generic in this context. These questions may be posed both for a general system (1) and for a specific system such as the forced harmonic oscillator

$$
\ddot{x}+x=\cos \omega t .
$$

A detailed discussion of the generic geometry of $V_{c}^{\prime}$ and its outline for the general system (1) and for the linear system (6) can be found in [16] where these questions are answered.

2.1.2. The re-set map $\phi_{c}$. There is a natural smooth re-set map $\phi_{c}: V_{c} \rightarrow \Pi$ obtained simply by re-setting the clock to $t=0$ at the moment of impact:

$$
\phi_{c}: V_{c} \rightarrow \Pi:(\tau, v ; t) \mapsto\left(\tau+t, \dot{x}_{c}(\tau, v ; t)\right) .
$$




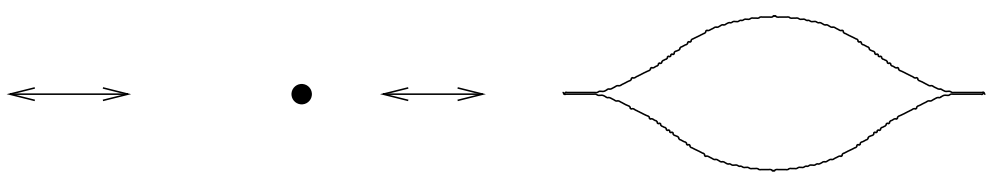

(a)
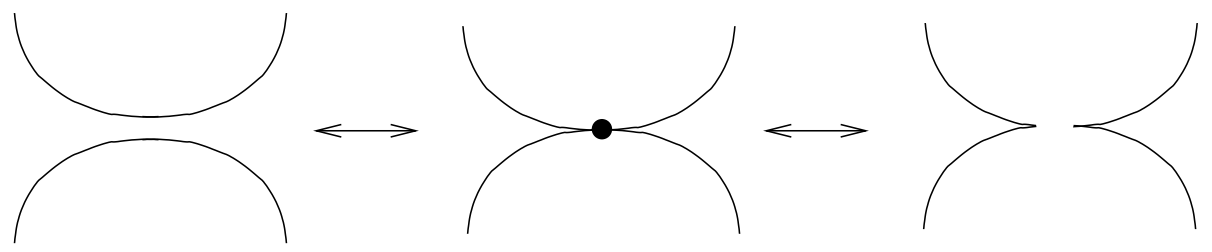

(b)
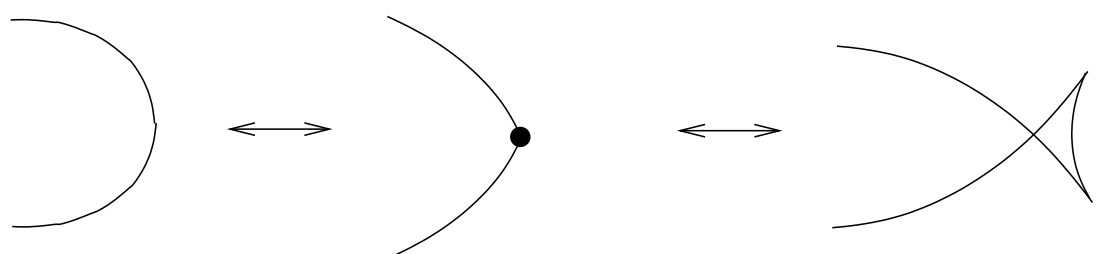

(c)

Figure 2. The three generic transitions in apparent outlines: (a) lips, (b) beaks, (c) swallowtail.

Let $Z_{c}$ denote the zero set of $V_{c}$, that is the intersection of $V_{c}^{\prime}$ with the plane $v=0$. The following facts about the singularity structure of $\phi_{c}$ are proved in [16, Propositions 6,7].

Proposition 2.2 The singular set of $\phi_{c}$ is $Z_{c} \backslash \Pi_{0}$. Points $(\tau, 0 ; t) \in Z_{c} \backslash \Pi_{0}$ where $a_{c}(\tau) \neq 0$ are fold points of $\phi_{c}$, while points where $a_{c}(\tau)=0$ but $a_{c}^{\prime}(\tau) \neq 0$ are cusp points.

2.1.3. The restitution map $R_{c}$. The restitution map describes how the velocity immediately after impact depends on the velocity immediately before impact. Thus we write

$$
\begin{array}{lll}
\Pi^{+}=\{(\tau, v) \in \Pi: v>0\}, & \Pi_{0}^{+}=\Pi^{+} \cup \Pi_{0} \\
\Pi^{-}=\{(\tau, v) \in \Pi: v<0\}, & \Pi_{0}^{-}=\Pi^{-} \cup \Pi_{0}
\end{array}
$$

and we suppose that $R_{c}: \Pi_{0}^{-} \rightarrow \Pi_{0}^{+}$is the restriction to $\Pi_{0}^{-}$of a map

$$
R_{c}: \Pi \rightarrow \Pi:(\tau, v) \mapsto\left(\tau, \rho_{c}(v)\right)
$$

where $\rho_{c}: \mathbf{R} \rightarrow \mathbf{R}$ is a differentiable function with $\rho_{c}(0)=0$ and $\rho_{c}^{\prime}(0)<0$. In many cases an appropriate choice is $\rho_{c}(v)=-r v$ with $0<r<1$ independent of $c$, although all that we shall require is that $\rho_{c}^{\prime}(0)=-r$.

\subsection{The first-hit map $F_{c}$ and the dynamical system $G_{c}$}

Since we are assuming the dynamics take place in the region $x \geq c$ it is only the upper half-plane $\Pi_{0}^{+}$that is of physical interest. Nevertheless, in much of what follows it will 
be useful first to consider $\Pi$ as a whole and then restrict to $\Pi_{0}^{+}$later.

The projection $p_{c}: V_{c} \rightarrow \Pi$ has a partial right inverse constructed as follows. Choose initial data $(\tau, v) \in \Pi^{+}$(so $x=c$ and $\dot{x}=v>0$ at $\left.t=0\right)$, then proceed parallel to the $t$-axis in the direction of increasing $t$ until the point $F_{c}(\tau, v)$ of first intersection (first hit) with $V_{c}$ is reached: thus

$$
F_{c}(\tau, v)=\left(\tau, v ; t_{1}\right) \in V_{c}^{\prime}
$$

where $(\tau, v ; t) \notin V_{c}$ for $0<t<t_{1}$. It is possible that no such $t_{1}$ exists (no future impacts occur), but for notational simplicity we disregard such points and write

$$
F_{c}: \Pi^{+} \rightarrow V_{c}^{\prime}
$$

in any case, with the understanding that $F_{c}$ may not be defined on the whole of $\Pi^{+}$.

The construction of $F_{c}$ extends naturally to points $(\tau, 0) \in \Pi_{0}$ for which $x_{c}(\tau, 0 ; t)>$ 0 for all sufficiently small $t>0$; if this does not hold (for example, if $\ddot{x}_{c}(\tau, 0 ; 0)<0$ ) then the constrained orbit remains stationary at $x=c$ and we define $F_{c}(\tau, 0)=(\tau, 0)$. The points of $V_{c}^{\prime}$ in the image of $F_{c}: \Pi_{0}^{+} \rightarrow V_{c}^{\prime}$ will be called visible points, as these are the points of $V_{c}^{\prime}$ that can be 'seen' from $\Pi_{0}^{+}$along the positive $t$ direction.

Finally, although it is not needed for dynamical purposes, for geometric convenience we define $F_{c}: \Pi^{-} \rightarrow V_{c}^{\prime}$ analogously, proceeding here in the direction of negative $t$.

The first-hit map $F_{c}: \Pi \rightarrow V_{c}^{\prime}$ defined in this way typically has discontinuities. Since $p_{c}$ is a local diffeomorphism at points $z \in V_{c}^{\prime} \backslash H_{c}$ it follows that the discontinuities of $F_{c}$ must lie in the apparent outline $P_{c}=p\left(H_{c}\right)$ or on $\Pi_{0}$. Not all points $w \in P_{c}$ are discontinuity points of $F_{c}$, of course, but only those with $F_{c}(w) \in H_{c}$ : thus in Figure 1 the point $w_{1}$ is a point of discontinuity of $F_{c}$ while the point $w_{3}$ is not.

\subsection{The correspondence principle}

The task of describing the dynamics of the impact oscillator close to grazing is greatly helped by exploiting a correspondence, induced naturally by $\phi_{c}$, between geometric features of $P_{c}$ close to $\Pi_{0}$ and those away from $\Pi_{0}$.

If $z \in H_{c}$ then $p_{c}(z) \in P_{c}$ by definition, and since $\dot{x}_{c}=0$ at $z$ we have $\phi_{c}(z) \in \Pi_{0}$. Likewise if $z \in Z_{c}$ then $p_{c}(z) \in \Pi_{0}$ by definition, and it is also the case that $\phi_{c}(z) \in P_{c}$. This is because $z$ represents current data (on $x=c$ ) for a solution with initial velocity $v=0$ so, by uniqueness of solutions of (1), after re-set the point $\phi_{c}(z)$ represents initial data for a solution $x(t)$ with $x(t)=c$ and with $\dot{x}(t)=0$ at some other time $t$. Thus

$$
\phi_{c}\left(H_{c}\right)=\Pi_{0}=p_{c}\left(Z_{c}\right) \quad \text { and } \quad p_{c}\left(H_{c}\right)=P_{c}=\phi_{c}\left(Z_{c}\right) .
$$

In fact by extending this argument we obtain a more general result which we leave as an exercise: 


\section{Proposition 2.3}

$$
p_{c}^{-1}\left(P_{c} \cup \Pi_{0}\right)=\phi_{c}^{-1}\left(P_{c} \cup \Pi_{0}\right) \text {. }
$$

Furthermore, since re-setting the time clearly does not affect geometric features of the dynamics, any features of the outline $P_{c}$ in a neighbourhood of $w=p_{c}(z) \in P_{c}$ where $z \in H_{c}$ must have counterparts in a neighbourhood of $\phi_{c}(z) \in \Pi_{0}$. This correspondence principle between $P_{c}$ and $\Pi_{0}$ enables us to focus the analysis on points of $\Pi_{0}$ when studying local dynamics close to grazing, and (as we see below) provides a valuable framework for understanding the structure of the dynamics itself. A fuller description of the geometry associated to the correspondence principle can be found in [16].

\subsection{Construction of the dynamics}

We are now ready to construct the (discontinuous) discrete dynamical system on the half-plane $\Pi_{0}^{+}$that we will use to model the dynamics of the impact oscillator.

Begin by applying the first-hit map $F_{c}: \Pi_{0}^{+} \rightarrow V_{c}^{\prime}$. This corresponds to following a solution of (1) as it leaves the obstacle at $x=c$ until it returns to $x=c$ for the first time. Next, apply the re-set map $\phi_{c}: V_{c}^{\prime} \rightarrow \Pi$; this is simply a re-calibration of the time variable. Since the initial data lie in $\Pi_{0}^{+}$the velocity at first hit must be negative or zero, so we have

$$
\phi_{c} \circ F_{c}: \Pi_{0}^{+} \rightarrow \Pi_{0}^{-} .
$$

Finally apply the restitution map $R_{c}: \Pi_{0}^{-} \rightarrow \Pi_{0}^{+}$and consider the composition:

$$
\Pi_{0}^{+} \stackrel{F_{c}}{\rightarrow} V_{c}^{\prime} \stackrel{\phi_{c}}{\longrightarrow} \Pi_{0}^{-} \stackrel{R_{c}}{\longrightarrow} \Pi_{0}^{+} .
$$

The iteration of this process reconstructs the impact dynamics of the oscillator as a (discontinuous) discrete dynamical system on $\Pi_{0}^{+}$generated by the map

$$
G_{c}:=R_{c} \circ \phi_{c} \circ F_{c}: \Pi_{0}^{+} \rightarrow \Pi_{0}^{+} .
$$

As indicated above, we may regard $F_{c}$ and $R_{c}$ and hence also $G_{c}$ as maps defined on the whole of $\Pi$ whenever this is convenient.

The rest of this paper is devoted to describing fully the generic behaviour of iterations of $G_{c}$ close to the $\tau$-axis $\Pi_{0}$, both for fixed clearance $c$ and as a 1-parameter family of dynamical systems when $c$ or another parameter is varied. We take the partial results of [14] further by giving a rigorous derivation of the dynamics for nondegenerate chatter at regular points of $\Pi_{0}$ (that is where $a_{c}(\tau) \neq 0$ ) and degenerate chatter at tangency points of $\Pi_{0}$ (where $a_{c}(\tau)=0$ but the derivative $a_{c}^{\prime}(\tau) \neq 0$ ). We also consider a yet more degenerate situation that generically arises only for certain values of the clearance $c$ at so-called swan points of $\Pi_{0}$ (where $a_{c}(\tau)=a_{c}^{\prime}(\tau)=0$ but $a_{c}^{\prime \prime}(\tau) \neq 0$ ), and we show how the dynamics unfold and undergo newly-created discontinuities in a 1-parameter bifurcation as $c$ passes through such values. The creation of new cusp 
points for $p_{c}$ gives rise to convoluted stable manifolds of nearby chatter points, with immediate implications for complicated global dynamical behaviour. Understanding the global structure of stable manifolds is one of the main challenges in formulating a dynamical theory for non-smooth systems such as impact oscillators.

\section{Nondegenerate chatter}

Let the clearance $c$ be fixed. To simplify notation in this section we temporarily write $V^{\prime}, F, G$ etc. for $V_{c}^{\prime}, F_{c}, G_{c}$ etc., although we retain $v_{c}$ for clarity and understand that $p$ denotes $p \mid V_{c}^{\prime}$. Using coordinates $(\tau, v)$ on $\Pi$ and $(\tau, t)$ on $V^{\prime}$, we identify a point $\left(\tau_{*}, 0 ; 0\right)$ of the $\tau$-axis $\Pi_{0}=\Pi \cap V^{\prime}$ with both $w_{*}=\left(\tau_{*}, 0\right) \in \Pi$ and $z_{*}=\left(\tau_{*}, 0\right) \in V^{\prime}$.

Let $w_{*}=\left(\tau_{*}, 0\right) \in \Pi_{0}$ be a regular point of $\Pi_{0}$, so that $a\left(\tau_{*}\right) \neq 0$. Since the 'free' dynamics take place in the region $x>c$, the behaviour of any solution that leaves $x=c$ with zero velocity but positive acceleration will not be susceptible to our local (lowvelocity) analysis close to $\Pi_{0}$ : therefore we assume $a_{*}:=a\left(\tau_{*}\right)<0$. On leaving $x=c$ with small positive velocity the solution to (1) will slow down, then possibly reverse and hit $x=c$ again after a short time. This is indeed the case as we see from (5): since $z_{*} \notin H$ the map $p: V^{\prime} \rightarrow \Pi$ is a local diffeomorphism at $z_{*}$ and therefore $F$ is also a local diffeomorphism at $w_{*}$. From Proposition 2.2 we have that $\phi: V^{\prime} \rightarrow \Pi$ is a local diffeomorphism at $w_{*}$ and so $G=R \circ \phi \circ F$ is (the restriction to $\Pi_{0}^{+}$of) a local diffeomorphism of $\Pi$ at $w_{*}$.

\subsection{Local linearisation along $\Pi_{0}$}

As a first approximation to the dynamics we linearise at the equilibrium point $w_{*} \in \Pi_{0}$. Since by definition

$$
G \circ p=R \circ \phi
$$

we have on differentiation

$$
D G(w) D p(z)=D R(u) D \phi(z)
$$

for $z \in V^{\prime}$ where $w=p(z)$ and $u=\phi(z)$, both in $\Pi$. Writing $\tau=\tau_{*}+\sigma$ we see from (5) that the derivative $D p\left(z_{*}\right)$ is

$$
D p\left(z_{*}\right):(\sigma, t) \mapsto\left(\sigma,-\frac{1}{2} a_{*} t\right) .
$$

Also, implicit differentiation of (3) shows that $\frac{\partial v_{c}}{\partial t}\left(z_{*}\right)=\frac{1}{2} a_{*}$ so that (see [16, Lemma 2])

$$
D \phi\left(z_{*}\right):(\sigma, t) \mapsto\left(\sigma+t, \frac{1}{2} a_{*} t\right) .
$$

Hence the derivative of the local diffeomorphism $G=R \circ \phi \circ F$ at $w_{*}$ is given by the $2 \times 2$ matrix

$$
D G\left(w_{*}\right)=\left(\begin{array}{cc}
1 & -\frac{2}{a}_{*} \\
0 & r
\end{array}\right)
$$


where $r=-\rho^{\prime}(0)$ is the restitution coefficient (compare [14]).

Eigenvectors of $D G\left(w_{*}\right)$ are $(1,0)$ with eigenvalue 1 (since the $\tau$-axis $\Pi_{0}$ is fixed) and $\left(2, a_{*}(1-r)\right)$ with eigenvalue $r$. Therefore, to first order, we have the picture as illustrated in Figure 3(a) (see also [14]): orbits of the local linearisation of the discrete dynamics $G$ approach $\Pi_{0}$ along directions with negative slope that tends to zero as $a_{*} \rightarrow 0$ or as $r \rightarrow 1$.

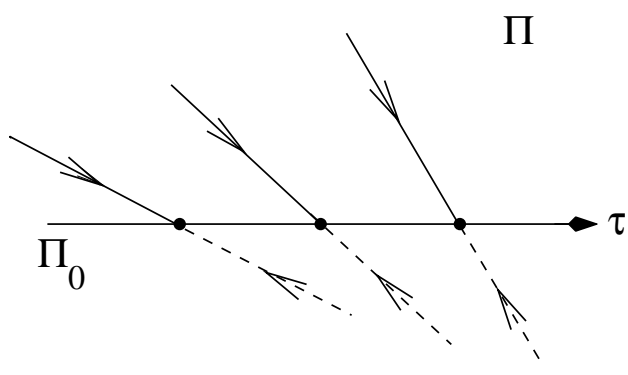

(a)

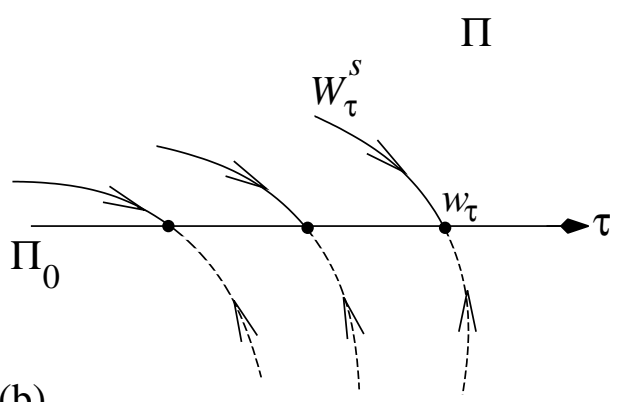

(b)

Figure 3. Local dynamics of $G$ in the $(\tau, v)$-plane $\Pi$ close to $\Pi_{0}$ : nondegenerate chatter for $a(\tau)<0$. (a) Local linearisation, (b) local foliation by stable manifolds.

This does not complete the story, however, as it remains to confirm that the linear description also accurately describes the nonlinear behaviour of $G$ on $\Pi$ close to $\Pi_{0}$.

A fundamental component in the theory of smooth dynamical systems is the analysis of dynamics in the neighbourhood of a normally hyperbolic invariant manifold, that is an invariant manifold for which the rate of normal contraction or expansion overpowers any contraction or expansion along the manifold itself: main references are [26] and [43]. See also [27] for an encyclopaedic treatment.

In our case, let $J$ be any open interval of the $\tau$-axis $\Pi_{0}$ on which $a(\tau)$ is negative and bounded away from zero. Then $G$ is the identity on $J$ and at each point $w \in J$ the derivative $D G(w)$ has a contracting eigenspace (since $0<r<1$ ) transverse to $\Pi_{0}$. Hence $J$ is a normally hyperbolic invariant manifold for $G$, and from the standard theory [26] we immediately deduce the following description of the local dynamics of $G$ on some neighbourhood $U$ of $J$ in $\Pi$. Recall, however, that for applications to the impact oscillator we are concerned only with forward iterations of $G$ and with the region $\Pi_{0}^{+}$, that is where $v \geq 0$.

Theorem 3.1 For each $\tau \in J$ there is a smooth $G$-invariant curve $W_{\tau}^{s}$ in $U \subset \Pi$ through $w_{\tau}=(\tau, 0) \in \Pi_{0}$ and tangent to the direction $(2,(1-r) a(\tau))$. The curve $W_{\tau}^{s}$ is a local stable manifold for $w_{\tau}$ :

$$
W_{\tau}^{s}=\left\{u \in U: G^{n}(u) \rightarrow w_{\tau} \text { as } n \rightarrow+\infty\right\} .
$$

The curves $\left\{W_{\tau}^{s}\right\}_{\tau \in J}$ provide a continuous foliation of $U$, in that there is a homeomorphism $\Phi: J \times \mathbf{R} \rightarrow U$ taking $\{\tau\} \times \mathbf{R}$ to $W_{\tau}^{s}$ for each $\tau \in J$. See Figure 3(b). 
Thus the linear picture is indeed an accurate representation of the nonlinear dynamics close to $\Pi_{0}$, although this result yields only that the stable manifold $W_{\tau}^{s}$ varies continuously with $\tau$. Can we obtain more, and see that in fact $W_{\tau}^{s}$ varies smoothly with $\tau$ so that the union of the $W_{\tau}^{s}$ form a smooth foliation of a neighbourhood of $\Pi_{0}$ ? From a result of Takens [43] the answer turns out to be positive.

Theorem 3.2 If the functions $f$ and $g$ in (1) are $C^{\infty}$ then (for suitable $U$ ) the homeomorphism $\Phi$ in Theorem 3.1 may be taken to be a $C^{\infty}$ diffeomorphism.

Proof. This follows from the more general (although local) theorem in [43] concerning partially hyperbolic fixed points. The non-resonance conditions which are imposed in the more general setting of [43] are in our case vacuously satisfied since $0<r<1$ and there is no expansion or contraction along the manifold $J$. Extension from the local result to the whole of $J$ follows by usual compactness arguments applied to the closure of $J$.

Therefore the dynamics of the local linearisation as described in [14] do indeed give an accurate picture of the true (nonlinear) dynamics up to smooth change of coordinates.

As $a(\tau) \rightarrow 0$ the stable manifold $W_{\tau}^{s}$ approaches $\Pi_{0}$ with slope tending to zero. Our next step is to investigate the local dynamics in this limiting case.

\section{Degenerate chatter}

In this section we examine the nature of chatter close to a point $w_{*}=\left(\tau_{*}, 0\right)$ where now $a_{c}\left(\tau_{*}\right)=0$. For generic choice of the functions $f$ and $g$ in (1) and for an open dense set of choices of the clearance $c$ the function $a_{c}(\tau)$ will have isolated zeros each with $a_{c}^{\prime}(\tau) \neq 0$. In particular this is the case for the linear system (6), since here $a_{c}(\tau)=-c+\cos \omega \tau$. However, for certain values of $c$ the function $a_{c}(\tau)$ can typically be expected to undergo Morse transitions (fold bifurcations) where $a_{c}(\tau)=0$ and $a_{c}^{\prime}(\tau)=0$ while $a_{c}^{\prime \prime}(\tau) \neq 0$. We shall describe the local dynamics of the impact oscillator in both these situations.

We start by calculating the first few terms of the Taylor series of the maps

$$
p_{c}:(\tau, t) \mapsto\left(\tau, v_{c}(\tau, t)\right)
$$

and

$$
\phi_{c}:(\tau, t) \mapsto\left(\tau+t, \dot{x}_{c}\left(\tau, v_{c}(\tau, t)\right)\right.
$$

at points $(\tau, 0) \in \Pi_{0}$. Since from (4) we have

$$
v=\dot{x}_{c}(\tau, v ; 0)=y_{c}(\tau, v ; 0)
$$

it follows that $\frac{\partial y_{c}}{\partial v}=1$ and all other derivatives of $y_{c}$ with respect to $v$ and/or $\tau$ vanish on $\Pi_{0}$. Using this, by implicit differentiation of (3) and writing $\tau=\tau_{*}+\sigma$ we find

$$
v_{c}\left(\tau_{*}+\sigma, t\right)=-\frac{1}{2} a t
$$




$$
\begin{aligned}
& -\frac{1}{2} a^{\prime} \sigma t+\frac{1}{2}\left(-\frac{1}{3} a^{\prime}+\frac{1}{2} a b\right) t^{2} \\
& -\frac{1}{4} a^{\prime \prime} \sigma^{2} t+\frac{1}{2}\left(-\frac{1}{3} a^{\prime \prime}+\frac{1}{2} a^{\prime} b\right) \sigma t^{2} \\
& \quad-\frac{1}{6}\left(\frac{1}{4} a^{\prime \prime}+\frac{3}{2}\left(-\frac{1}{3} a^{\prime}+\frac{1}{2} a b\right) b\right) t^{3}+O(4)
\end{aligned}
$$

where $a=a_{c}\left(\tau_{*}\right), b=\frac{\partial}{\partial v} \ddot{x}_{c}\left(\tau_{*}, 0 ; 0\right)$ and the primes denote derivatives with respect to $\tau$ evaluated at $\tau_{*}$ (which in general depend also on $c$ ). Hence, if $a \neq 0$ then

$$
v_{c}\left(\tau_{*}+\sigma, t\right)=-\frac{1}{2} a t+O(2)
$$

while if $a=0$ but $a^{\prime} \neq 0$ then

$$
v_{c}\left(\tau_{*}+\sigma, t\right)=-\frac{1}{6} a^{\prime} t(3 \sigma+t)+O(3) .
$$

Finally, if $a=a^{\prime}=0$ but $a^{\prime \prime} \neq 0$ then

$$
v_{c}\left(\tau_{*}+\sigma, t\right)=-\frac{1}{2} 4 a^{\prime \prime} t\left(6 \sigma^{2}+4 \sigma t+t^{2}\right)+O(4) .
$$

\subsection{Dynamics near a tangency point}

A point $(\tau, 0) \in \Pi_{0}$ where $a_{c}(\tau)=0$ and $a_{c}^{\prime}(\tau) \neq 0$ is called a tangency point. In the context of Section 3 this can be regarded as a point of $\Pi_{0}$ where the normal hyperbolicity fails in the least degenerate way.

Suppose that $w_{*}=\left(\tau_{*}, 0\right) \in \Pi_{0}$ is a tangency point. From (16) we see that $v_{c}$ has a saddle critical point at $(\sigma, t)=(0,0)$. The horizon $H_{c}$ is tangent to the line $3 \sigma+2 t=0$ which projects from $V_{c}^{\prime}$ by $p_{c}$ to the graph in $\Pi$ of a function

$$
v=\frac{3}{8} a_{c}^{\prime}\left(\tau_{*}\right) \sigma^{2}+O(3) .
$$

We consider two cases separately, according to the sign of $a_{c}^{\prime}\left(\tau_{*}\right)$. Again for convenience we drop the suffix $c$ from the notation.

(a) The case $a^{\prime}\left(\tau_{*}\right)>0$

The points of $H$ close to $w_{*}$ have $v \geq 0$. The part of $H$ relevant for us is the arc where $t \geq 0$, that is $\sigma \leq 0$, and so the relevant part of $P=p(H)$ here is a smooth arc $P_{*}$ quadratically tangent to $\Pi_{0}$ at $w_{*}$ from the direction $\tau<\tau_{*}$ and $v>0$. This is the discontinuity set for the first-hit map $F$ and hence for $G$ locally: we call it the discontinuity arc. See Figure 4.

Theorem 4.1 Let $w_{*}=\left(\tau_{*}, 0\right)$ be a tangency point with $a^{\prime}\left(\tau_{*}\right)>0$. There is a smooth arc $\gamma \subset \Pi_{0}^{+}$with an end-point at $w_{*}$, quadratically tangent to $\Pi_{0}$ at $w_{*}$ from the side $\tau<\tau_{*}$ and otherwise lying between $P_{*}$ and $\Pi_{0}$. The arc $\gamma$ is forward invariant for $G$ (that is $G(\gamma) \subset \gamma$ ) and is locally unique in the sense that if $\gamma, \gamma^{\prime}$ are two such arcs then one is a subset of the other. The fixed point $w_{*}$ is exponentially attracting for $G \mid \gamma$, while $\gamma$ is weakly normally hyperbolic (repelling) for $G$ near $w_{*}$. Orbits close to but not on $\gamma$ either (if below $\gamma$ ) are attracted exponentially to $\Pi_{0}$ in nondegenerate chatter or (if above $\gamma$ ) cross the discontinuity arc $P_{*}$ after finitely many iterations of $G$, corresponding to loss of immediate contact with the obstacle. See Figure 5. 
By weakly normally hyperbolic near $w_{*}$ we mean that at points $w \in \gamma$ the derivative $D G(w)$ has an eigenvalue $\lambda \neq 1$ (in this case $\lambda>1$ ) with eigendirection transverse to $\gamma$, although $\lambda \rightarrow 1$ as $w \rightarrow w_{*}$ along $\gamma$. The terms above and below correspond to values of $v$.

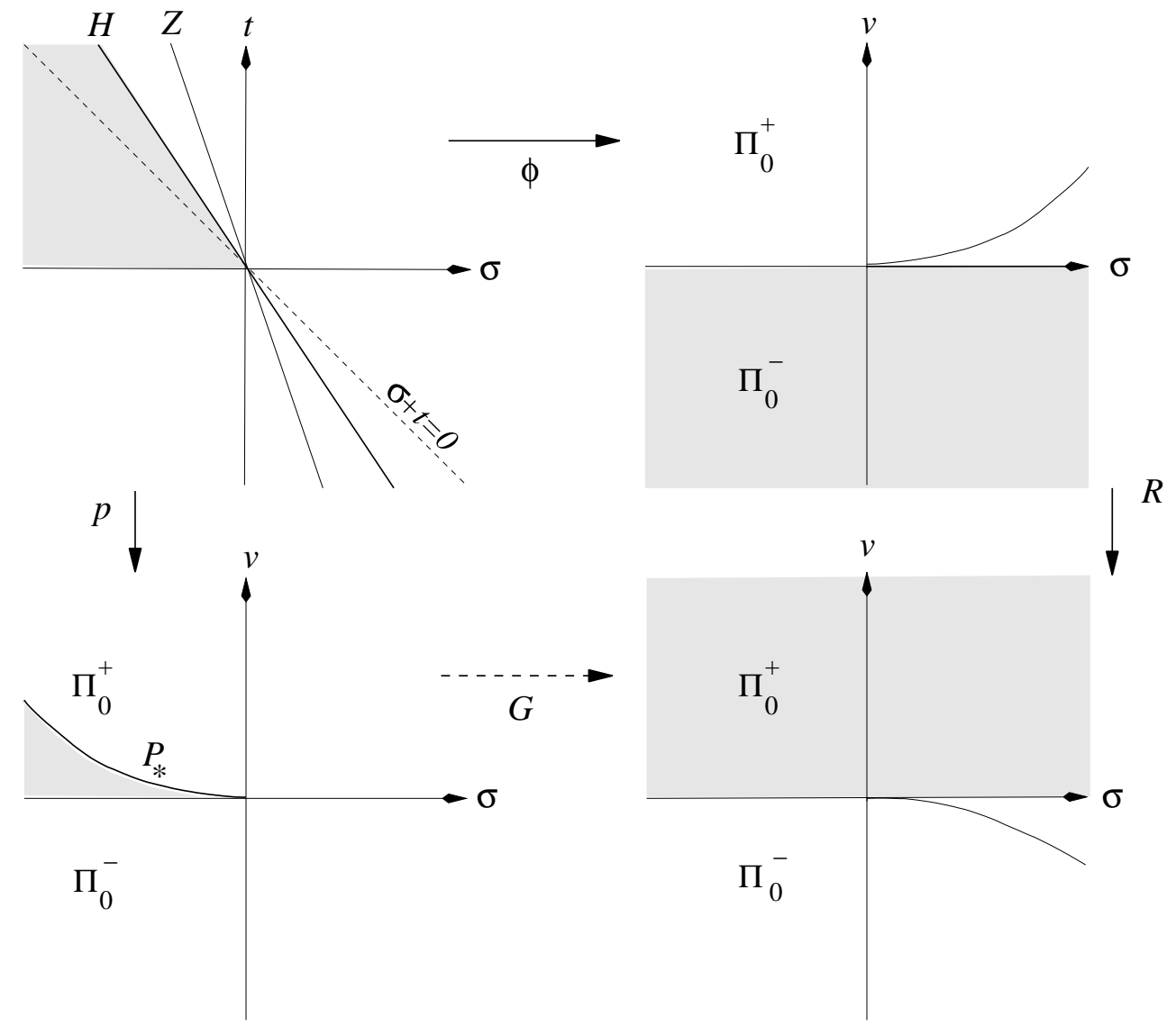

Figure 4. The actions of $p, \phi$ and $R$ close to $\left(\tau_{*}, 0\right)$ with $a^{\prime}\left(\tau_{*}\right)>0$ : here $\tau=\tau_{*}+\sigma$. The shaded area in the top left diagram represents the visible part of $V^{\prime}$, and the other shaded areas are its images under the relevant maps as indicated. The $\operatorname{arc} P_{*} \subset p(H)$ is the discontinuity arc.

A proof of the existence of such an arc $\gamma$, approximately forward invariant up to second order, was given by Budd and Dux in [14]. The analysis in [14] is also for a particular linear system, although the authors observe that their results apply more generally. We provide here a complete proof that applies in full generality to nonlinear systems of type (1).

4.1.1. Proof of Theorem 4.1. From (8) and (16) we see that the projection $p_{c}: V^{\prime} \rightarrow \Pi$ is given in local coordinates by

$$
p:(\sigma, t) \mapsto\left(\sigma,-k\left(3 \sigma t+t^{2}\right)+O(3)\right)
$$

where $k=\frac{1}{6} a^{\prime}\left(\tau_{*}\right)>0$, and from (9) and (16) the reset map $\phi: V^{\prime} \rightarrow \Pi$ is given by

$$
\phi:(\sigma, t) \mapsto\left(\sigma+t,-k\left(3 \sigma t+2 t^{2}\right)+O(3)\right) .
$$


Figure 4 indicates some local geometry of $p$ and of $\phi$. As already noted, to first order the horizon $H$ is given by $3 \sigma+2 t=0$, while to first order the zero set $Z$ is given by $3 \sigma+t=0$. Also, the line $\sigma+t=0$ is by definition taken by $\phi$ to the $v$-axis $\sigma=0$.

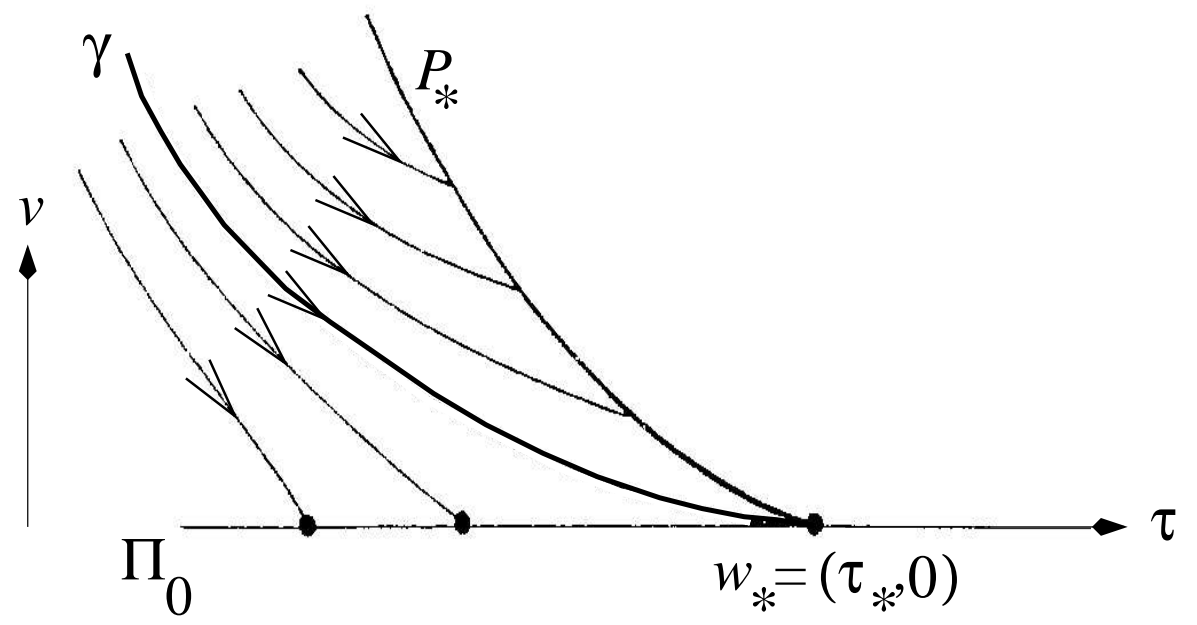

Figure 5. Degenerate chatter at $w_{*}=\left(\tau_{*}, 0\right)$ with $a^{\prime}\left(\tau_{*}\right)>0$. Invariant curves below the invariant curve $\gamma$ represent nondegenerate chatter; invariant curves above $\gamma$ carry orbits across the discontinuity $\operatorname{arc} P_{*}$.

Now take new coordinates $(\sigma, \eta)$ in $\mathbf{R}^{2}$, where $\eta=\frac{3}{2} \sigma+t$. Then

$$
p:(\sigma, \eta) \mapsto\left(\sigma,-k \eta^{2}+\frac{9}{4} k \sigma^{2}+O(3)\right) .
$$

Composing with the diffeomorphism

$$
\psi: \mathbf{R}^{2} \rightarrow \mathbf{R}^{2}:(u, w) \mapsto\left(u, w-\frac{9}{4} k u^{2}\right)
$$

we obtain

$$
\psi \circ p:(\sigma, \eta) \mapsto\left(\sigma,-k \eta^{2}+O(3)\right)
$$

which exhibits $p$ explicitly as a fold map. By standard methods of singularity theory (see e.g. Arnol'd et al. [4], Golubitsky et al. [22]), after composing with local $C^{\infty}$ diffeomorphisms of $\mathbf{R}^{2}$ in range and domain that are the identity to second order, we may in fact assume that in a neighbourhood of $(0,0)$ we have

$$
\psi \circ p:(\sigma, \eta) \mapsto\left(\sigma,-k \eta^{2}\right)
$$

without any higher order terms.

Let $q: V^{\prime} \rightarrow \Pi$ denote the composition $R \circ \phi$. In the new $(\sigma, \eta)$ coordinates we find

$$
\psi \circ q:(\sigma, \eta) \mapsto\left(\eta-\frac{1}{2} \sigma,-r k\left(2 \eta^{2}-3 \sigma \eta\right)-\frac{9}{4} k\left(\eta-\frac{1}{2} \sigma\right)^{2}+O(3)\right)
$$


where we recall that $R(\tau, v)=(\tau, \rho(v))$ and $r=-\rho^{\prime}(0)$.

To say that $\gamma$ is an invariant curve for $G$ is equivalent to saying that the curve $\beta=F(\gamma) \subset V^{\prime}$ has the property that $p(\beta)=q(\beta)$, which in turn is equivalent to saying that $\beta$ is an invariant curve for the map $h:=p^{-1} \circ q$ whose domain and range are subsets of $V^{\prime}$. Explicitly we have

$$
h=p^{-1} \circ q:(\sigma, \eta) \mapsto\left(\eta-\frac{1}{2} \sigma, \sqrt{Q_{r}(\sigma, \eta)+O(3)}\right)
$$

where

$$
Q_{r}(\sigma, \eta):=r\left(2 \eta^{2}-3 \sigma \eta\right)+\frac{9}{4}\left(\eta-\frac{1}{2} \sigma\right)^{2} .
$$

Our strategy for finding $\beta$ will be to blow up the coordinates at $(\sigma, \eta)=(0,0)$ and then exhibit $\beta$ in the new coordinates as the stable manifold of a hyperbolic fixed point of $G$. For the blow-up construction we write

$$
(\sigma, \eta)=(s, s m)=: \chi(s, m)
$$

so that $m$ is the slope of the ray from the origin on which $(\sigma, \eta)$ lies, and $s$ measures displacement along the ray. Then

$$
h(s, s m)=\left(\left(m-\frac{1}{2}\right) s, \sqrt{s^{2} Q_{r}(1, m)+O\left(s^{3}\right)}\right)
$$

and so using $(s, m)$ as coordinates this becomes

$$
h^{\prime}:(s, m) \mapsto\left(s^{\prime}, m^{\prime}\right)
$$

where

$$
\begin{aligned}
& s^{\prime}=\left(m-\frac{1}{2}\right) s \\
& m^{\prime}=\left(m-\frac{1}{2}\right)^{-1} \sqrt{Q_{r}(1, m)+O(s)} .
\end{aligned}
$$

The domain $D_{r}$ of $h^{\prime}$ is the subset of $\mathbf{R}^{2}$ corresponding to the values of $m$ for which $m \neq \frac{1}{2}$ and the expression under the square root is nonnegative, for $|s|$ sufficiently small. The following is straightforward to check.

Proposition 4.2 The quadratic function $m \mapsto Q_{r}(1, m)$ has zeros at $m_{-}, m_{+}$where

$$
0<m_{-}<\frac{1}{2}<m_{+}<\frac{3}{2}
$$

and $Q_{r}(1, m)>0$ for $m \in E_{r}:=\left(-\infty, m_{-}\right] \cup\left[m_{+}, \infty\right)$. Also $m_{-}, m_{+} \rightarrow \frac{1}{2}$ as $r \rightarrow 0$.

Since two distinct zeros of a quadratic function are necessarily simple zeros, the Implicit Function Theorem yields the following result.

Corollary 4.3 For sufficiently small $\epsilon>0$ the domain $D_{r}$ of $h^{\prime}$ contains the region $D_{r}(\epsilon)=D_{r}^{-}(\epsilon) \cup D_{r}^{+}(\epsilon)$ where

$$
\begin{aligned}
& D_{r}^{-}(\epsilon):=\left\{(s, m) \in \mathbf{R}^{2}:|s|<\epsilon, m<\delta_{-}(s)\right\}, \\
& D_{r}^{+}(\epsilon):=\left\{(s, m) \in \mathbf{R}^{2}:|s|<\epsilon, m>\delta_{+}(s)\right\}
\end{aligned}
$$

and $\delta_{ \pm}$are smooth functions with $\delta_{ \pm}(0)=m_{ \pm}$respectively. 
We now seek a fixed point for $h^{\prime}$ on $0 \times E_{r}$. This occurs where

$$
m=\left(m-\frac{1}{2}\right)^{-1} \sqrt{Q_{r}(1, m)},
$$

that is where

$$
Q_{r}(1, m)=m^{2}\left(m-\frac{1}{2}\right)^{2} .
$$

Solutions of (28) are given by $m=\frac{3}{2}$ and by the solutions of

$$
2 r m=\left(m-\frac{1}{2}\right)^{2}\left(m+\frac{3}{2}\right) .
$$

A simple sketch of the graph of the cubic function on the right hand side of (29) shows that this equation has three solutions $m_{1}, m_{2}, m_{3}$ with

$$
m_{1}<-\frac{3}{2} \quad \text { and } \quad 0<m_{2}<\frac{1}{2}<m_{3} .
$$

We now investigate these four solutions of (28) and verify that only one of them is relevant here.

Solution $m=\frac{3}{2}$ : in the original $(\sigma, t)$ coordinates this corresponds to $t=0$, that is the $\tau$-axis $\Pi_{0}$. Clearly this is invariant under $G$ (on one side of $\left(\tau_{*}, 0\right)$ ) but it represents equilibrium states and is not the arc $\gamma$ representing chatter that we seek. In fact $m=\frac{3}{2}$ implies $r=1$ which is the ideal case of perfect restitution in the limit as $v \rightarrow 0$.

Solution $m_{1}<-\frac{3}{2}$ : the image of this line is given opposite orientations by $p$ and $q$, so that under the composition $q \circ p^{-1}$ the orientation of the image is reversed. Such a curve cannot be a candidate for $\gamma$. Moreover, since the gradient of the line is less than -3 it maps under $p$ (or $q$ ) to a curve tangent to the $\tau$-axis but with $v \leq 0$, not relevant here.

Solution $0<m_{2}<\frac{1}{2}$ : the image of this line is also given opposite orientations by $p$ and $q$ (in particular, points with $v>0$ are taken to points with $v<0$ ) so this image cannot be a candidate for $\gamma$.

Solution $m_{3}>\frac{1}{2}$ : here when $t>0$ both $p$ and $q$ take this line into the region $v>0$ with the same orientation, so if $\gamma=p(\beta)$ exists as an invariant curve then the tangent direction to $\beta$ at the origin must correspond to this ray.

Note that $m_{3}$ increases from $\frac{1}{2}$ to $\frac{3}{2}$ as $r$ increases from 0 to 1 .

We next study local linearisation of $h^{\prime}$ at $\left(0, m_{3}\right)$. Using $(22)$ and $(23)$ we find that the Jacobian matrix for $h^{\prime}$ at a point $(0, m)$ has the form

$$
D h^{\prime}(0, m)=\left(\begin{array}{cc}
m-\frac{1}{2} & 0 \\
* & N_{r}(m)
\end{array}\right)
$$

with

$$
\begin{aligned}
N_{r}(m) & =Q^{-\frac{1}{2}}\left(\frac{1}{2}\left(m-\frac{1}{2}\right)^{-1}\left[r(4 m-3)+\frac{9}{2}\left(m-\frac{1}{2}\right)\right]-Q\left(m-\frac{1}{2}\right)^{-2}\right) \\
& =r(2 m+3)(2 m-1)^{-2} Q^{-\frac{1}{2}}
\end{aligned}
$$


where $Q$ stands for $Q_{r}(1, m)$ and where $*$ denotes a term which depends on the $O(3)$ term in $\phi$. In the case when $m$ satisfies the fixed point equation (28) this further simplifies (eliminating $r$ ) to give

$$
N_{r}(m)=\frac{(2 m+3)^{2}}{8 m^{2}(2 m-1)} .
$$

As the eigenvalues of $D h^{\prime}(0, m)$ are $\lambda=\left(m-\frac{1}{2}\right)$ and $\lambda^{\prime}=N_{r}(m)$ we easily conclude the following.

Proposition 4.4 The fixed point $m_{3}$ with $\frac{1}{2}<m_{3}<\frac{3}{2}$ has eigenvalues $\lambda, \lambda^{\prime}$ with $0<\lambda<1$ and $\lambda^{\prime}>1$. Also

$$
\lambda \rightarrow 1, \quad \mu \rightarrow 1 \quad \text { as } \quad r \rightarrow 1 \quad\left(m_{3} \rightarrow \frac{3}{2}\right)
$$

while

$$
\lambda \rightarrow 0, \quad \mu \rightarrow \infty \quad \text { as } \quad r \rightarrow 0 \quad\left(m_{3} \rightarrow \frac{1}{2}\right) .
$$

The eigendirection for $\mu$ is the m-axis.

Note that the eigendirection for $\lambda$ would be the $s$-axis if there were no $O(3)$ terms in $\phi$.

Since $\left(0, m_{3}\right)$ is a hyperbolic fixed point for $h^{\prime}$ it has a unique (local) smooth stable manifold $W^{s}$ transverse to the $m$-axis. Then $\chi\left(W^{s}\right)$ is a smooth curve $\beta$ through the origin in $\mathbf{R}^{2}$ and tangent to the $s$-axis from above $(\eta>0)$ or below $(\eta<0)$ according as $\sigma<0$ or $\sigma>0$, and the same holds in the original $(\sigma, t)$ coordinates. By construction, since $h^{\prime}\left(W^{s}\right) \subset W^{s}$ then $h(\beta) \subset \beta$ which in turn implies forward invariance of $\gamma:=p(\beta) \cap \Pi_{0}^{+}$under $G$. The dynamical properties of $h^{\prime}$ near the saddle point $\left(0, m_{3}\right)$ carry over via $\chi$ to the stated properties of $G$ on and close to $\gamma$. This completes the proof of Theorem 4.1.

(b) The case $a^{\prime}\left(\tau_{*}\right)<0$

Here the horizon $H$ for $(\tau, t) \neq\left(\tau_{*}, 0\right)=w_{*}$ locally lies in the region $v<0$ and so $F: \Pi_{0}^{+} \rightarrow V^{\prime}$ and hence also $G: \Pi_{0}^{+} \rightarrow \Pi_{0}^{+}$are continuous maps in a neighbourhood of $w_{*}$ in $\Pi_{0}^{+}$. Moreover, in a neighbourhood of the origin $G$ is a homeomorphism onto its image and is differentiable away from $w_{*}$. In detail, with local coordinate $\tau=\tau_{*}+\sigma$ as before, we see that to first order $F$ maps a neighbourhood $\mathcal{T}^{\prime}$ of the origin in $\Pi_{0}^{+}$onto a neighbourhood of the origin in the sector

$$
\mathcal{T}: \quad 3 \sigma+t \geq 0, t \geq 0
$$

in $\mathbf{R}^{2}$ (coordinates on $V^{\prime}$ ) which is then taken by $\phi$ to a neighbourhood of the origin in the region $\mathcal{T}^{\prime \prime}$ bounded by the positive $\sigma$-axis and an arc tangent to it given by

$$
v=\frac{3}{8} a^{\prime}\left(\tau_{*}\right) \sigma^{2}+O(3)
$$

in $\Pi_{0}^{-}$: see Figure 6. Finally, $R$ maps $\mathcal{T}^{\prime \prime}$ to a similar if narrower region $R \mathcal{T}^{\prime \prime}$ in $\Pi_{0}^{+}$. From this geometry it is reasonable to suppose that under forward iteration of $G$ all 


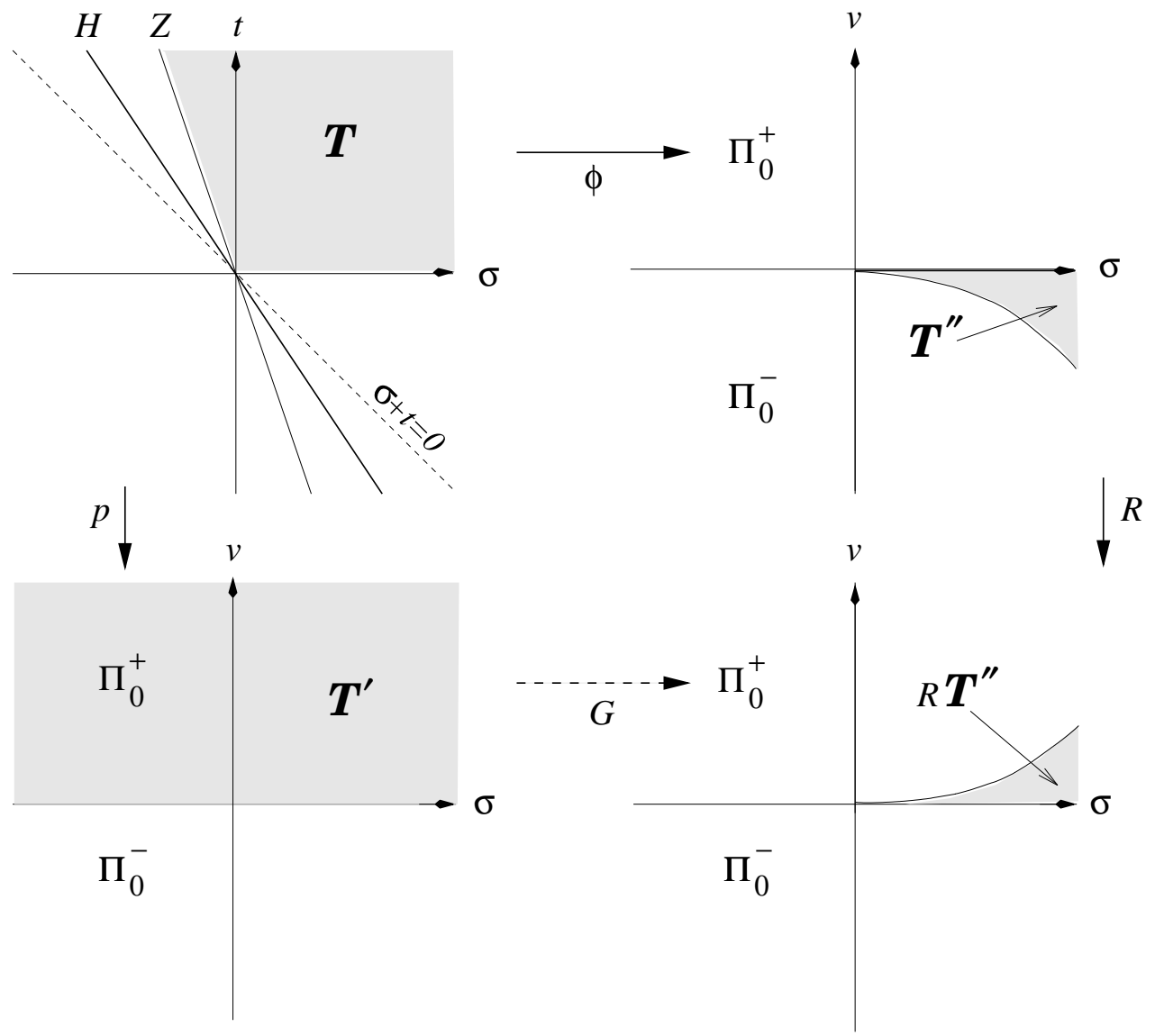

Figure 6. The actions of $p, \phi$ and $R$ close to $\left(\tau_{*}, 0\right)$ with $a^{\prime}\left(\tau_{*}\right)<0$ : here $\tau=\tau_{*}+\sigma$. The shaded area in the top left diagram represents the visible part of $V^{\prime}$, and the other shaded areas are its images under the relevant maps as indicated.

points of $\mathcal{T}^{\prime}$ tend to the $\sigma$-axis $\Pi_{0}$. We now look more closely to verify that this is the case.

First consider an arc $\alpha_{\ell}$ in $V^{\prime}$ given in $(\sigma, t)$ coordinates by $t=\ell \sigma$ with $\ell \geq 0$ and $\sigma \geq 0$. From (18) and (19) we have

$$
\begin{aligned}
& p(\sigma, \ell \sigma)=\left(\sigma,-k\left(3 \ell+\ell^{2}\right) \sigma^{2}+\ell \sigma^{3} \mu(\ell, \sigma)\right) \\
& q(\sigma, \ell \sigma)=\left(\sigma(1+\ell),-r k\left(3 \ell+2 \ell^{2}\right) \sigma^{2}+\ell \sigma^{3} \nu(\ell, \sigma)\right)
\end{aligned}
$$

where $\mu, \nu$ are continuous (in fact smooth) functions for $(\ell, \sigma)$ sufficiently small. The images of $\alpha_{\ell}$ under $p$ and $q$ are locally the graphs of two smooth functions $v=\tilde{p}_{\ell}(\sigma)$ and $v=\tilde{q}_{\ell}(\sigma)$ for $\sigma \geq 0$. Given constants $K>0, L>0$ we can choose $\delta>0$ such that

$$
\tilde{p}_{\ell}(\sigma)>-k \ell(3+\ell)(1-K) \sigma^{2}
$$

and

$$
\tilde{q}_{\ell}(\sigma)<-r k \ell(3+2 \ell)(1+\ell)^{-2}(1+L) \sigma^{2}
$$


for $0 \leq \ell \leq \delta$ and $0 \leq \sigma \leq \delta$, where as before $k=\frac{1}{6} a^{\prime}\left(\tau_{*}\right)$ and here $k<0$. It then follows that

$$
\frac{\tilde{q}_{\ell}(\sigma)}{\tilde{p}_{h \ell}(\sigma)}<\frac{3+2 \ell}{(1+\ell)^{2}(3+h \ell)} \frac{1+L}{1-K} \frac{r}{h}
$$

for any $0<h<1$. Choose $K, L$ sufficiently small (and $\delta$ accordingly) so that $c:=\frac{1+K}{1-L} r<1$. Since $(3+2 \ell)<\left(1+\ell^{2}\right)(3+h \ell)$ for all $\ell>0$ and $h>0$, the above estimates show that when $\ell, \sigma<\delta$ the $\operatorname{arc} F \circ R \circ \phi\left(\alpha_{\ell}\right)$ lies below the arc $\alpha_{h \ell}$ in $\mathcal{T}$ when $c<h<1$. In other words, when viewed in blown-up $\sigma, \ell$ coordinates in $\mathcal{T}$ the dynamics are dominated by exponential contraction in the $\ell$-coordinate.

A similar argument applies to the derivatives $\tilde{p}_{\ell}^{\prime}, \tilde{q}_{\ell}^{\prime}$ of $\tilde{p}_{\ell}, \tilde{q}_{\ell}$ with respect to $\sigma$ : for sufficiently small $\delta$ we can ensure that if $0 \leq \ell, \sigma \leq \delta$ then there exists $0<h<1$ such that $\tilde{q}_{\ell}^{\prime}(\sigma)<\tilde{p}_{h \ell}^{\prime}(\sigma)$ for $0<\sigma<\delta$, and that therefore $\tilde{p}_{\ell}(\sigma)$ tends $C^{1}$-uniformly to zero for $0 \leq \sigma \leq \delta$. Thus we obtain the anticipated result:

Proposition 4.5 Under iteration of $G$ the images of the arc $p\left(\alpha_{\ell}\right)$ tend $C^{1}$-uniformly to the $\tau$-axis over the interval $[0, \delta]$.

Using this fact and the results from Section 3 we can give a complete description of the local dynamics around the tangency point $w_{*}$. Let $D^{+}$denote the closed half-disk in $\mathbf{R}^{2}$ given by $D^{+}=\left\{(x, y) \in \mathbf{R}^{2}: x^{2}+y^{2} \leq 1, y \geq 0\right\}$, and let $E: D^{+} \rightarrow D^{+}$be the map described in polar coordinates $(x, y)=(\rho \cos \theta, \rho \sin \theta)$ by $\theta \mapsto \frac{1}{2} \theta$. The dynamics of $E$ are a topological model for the dynamics of $G$ close to $w_{*}$. More precisely:

Theorem 4.6 There is a homeomorphism $\Psi$ from $D^{+}$onto a closed neighbourhood $U$ of $w_{*}$ in $\Pi_{0}^{+}$with $\Psi(0,0)=w_{*}$ and such that $\Psi$ is a topological conjugacy between $E$ and $G$, that is $\Psi \circ E=G \circ \Psi$. Moreover, $\Psi$ can be chosen to be $C^{\infty}$ off the $x$-axis. In particular, $U$ is smoothly foliated (except perhaps on $\Pi_{0}$ ) by arcs of stable manifolds of points $w=(\tau, 0) \in \Pi_{0}$ with $\tau>\tau_{*}$, each arc having end-points on $\Pi_{0}$ on opposite sides of $\tau_{*}$. See Figure 7 .

Proof. Choose positive numbers $0<\delta_{1}<\delta \in \mathbf{R}$ where $\delta$ is small enough to satisfy the conditions imposed in the proof of Proposition 4.5. By Theorem 3.2 there is a neighbourhood in $\Pi_{0}^{+}$of the interval $J:=\left[w_{*}+\delta_{1}, w_{*}+\delta\right] \subset \Pi_{0}$ that is smoothly foliated by stable manifolds of points of $J$. By Proposition 4.5 there is some positive integer $n$ and interval $J^{\prime}:=\left[w_{1}^{\prime}, w_{*}\right] \subset \Pi_{0}$ such that $G^{n}\left(J^{\prime}\right)$ is sufficiently $C^{1}$-close to $J$ that it intersects the stable manifolds transversely. Let $G^{n}\left(w_{1}^{\prime}\right) \in W^{s}\left(w_{1}\right), w_{1} \in \Pi_{0}$. It follows by backward iteration of $G$ that the stable manifold of each $w \in J$ contains a closed arc with end-points on opposite sides of $w_{*}$ in $\Pi_{0}$, and in particular $W^{s}\left(w_{1}\right)$ contains an arc from $w_{1}^{\prime}$ to $w_{1}$. Since this construction applies for arbitrary $\delta_{1}$ with $0<\delta_{1} \leq \delta$ we obtain a neighbourhood of $w_{*}$ in $\Pi_{0}^{+}$smoothly foliated (except perhaps on $\left.\Pi_{0}\right)$ by stable manifolds as claimed.

To construct a conjugacy $\Psi$ we first construct a homeomorphism $\psi$ from the sector $D(n)$ of $D^{+}$given by $\frac{\pi}{2^{n+1}} \leq \theta \leq \frac{\pi}{2^{n}}$ to the region in $\Pi_{0}^{+}$bounded by an arc of $G^{n+1}\left(J^{\prime}\right)$, and an arc of $G^{n}\left(J^{\prime}\right)$ together with an arc of $W^{s}\left(w_{1}\right)$. This can be done in such a way 
that arcs in $D^{+}$of constant distance from the origin are taken by $\psi$ to arcs of stable manifolds of points in $\left[w_{*}, w_{1}\right]$. The homeomorphism $\psi$ is then extended to $\Psi$ on the whole of $D^{+}$except for the positive $x$-axis by $\Psi(u)=G^{-\ell} \circ \psi \circ E^{\ell}(u)$ where $\ell$ is chosen to be the unique integer (positive or negative) such that $E^{\ell}(u) \in D(n)$. With care, $\psi$ can be constructed at the boundary of $D(n)$ in such a way that $\Psi$ is a diffeomorphism away from the origin. Finally, $\Psi$ extends to the positive $x$-axis $(\theta=0)$ by continuity.

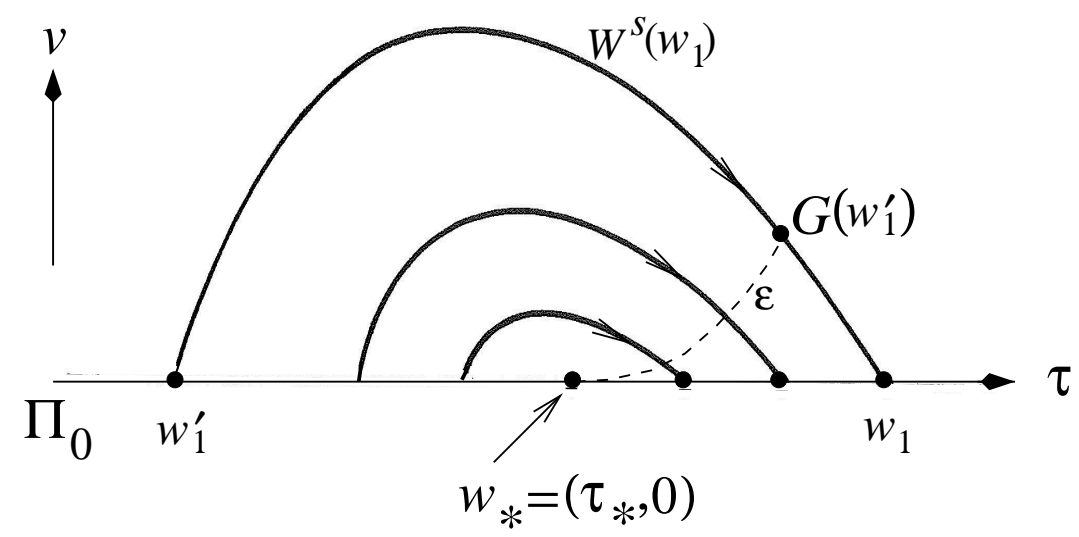

Figure 7. Chatter close to $w_{*}=\left(\tau_{*}, 0\right)$ with $a^{\prime}\left(\tau_{*}\right)<0$. Points of $\Pi_{0}$ with $\tau>\tau_{*}$ are limit points of nondegenerate chatter that becomes increasingly degenerate as $\tau \rightarrow \tau_{*}$ from above. The arc $\left[w_{1}^{\prime}, w_{*}\right]$ on $\Pi_{0}$ is taken by $G$ to an arc $\varepsilon$ tangent to $\Pi_{0}$ at $w_{*}$.

\section{Further degeneracy: dynamics near a swan point}

Finally we consider the dynamics of $G$ close to a swan point $\left(\tau_{*}, 0\right)$; here $a\left(\tau_{*}\right)=a^{\prime}\left(\tau_{*}\right)=$ 0 but $a^{\prime \prime}\left(\tau_{*}\right) \neq 0$.

As before, we work with coordinates $\tau=\tau_{*}+\sigma$ and we write $(\sigma, \eta)=(s, s m)=$ $\chi(s, m)$. Using (17) we see that the maps $p$ and $\phi$ then become

$$
\begin{aligned}
& p:(s, m) \mapsto\left(s, \ell s^{3}\left(6 m+4 m^{2}+m^{3}+O(s)\right)\right) \\
& \phi:(s, m) \mapsto\left(s(1+m),-\ell s^{3}\left(6 m+8 m^{2}+3 m^{3}+O(s)\right)\right)
\end{aligned}
$$

where $\ell=-\frac{1}{2} 4 a^{\prime \prime}\left(\tau_{*}\right)$ and where the terms $O(s)$ denote smooth functions of $(s, m)$ that vanish when $s=0$.

In $(s, m)$ coordinates the map $h$ becomes $\bar{h}:(s, m) \mapsto(\bar{s}, \bar{m})$ where

$$
\begin{aligned}
s(1+m) & =\bar{s} \\
r s^{3}\left(6 m+8 m^{2}+3 m^{3}+O(s)\right) & =\bar{s}^{3}\left(6 \bar{m}+4 \bar{m}^{2}+\bar{m}^{3}+O(s)\right)
\end{aligned}
$$

and substituting the first equation into the second for $s \neq 0$ we obtain

$$
r\left(6 m+8 m^{2}+3 m^{3}\right)=(1+m)^{3}\left(6 \bar{m}+4 \bar{m}^{2}+\bar{m}^{3}\right)+O(s) .
$$


The graphs of the two real-valued functions

$$
\begin{aligned}
& f: m \mapsto r\left(6 m+8 m^{2}+3 m^{3}\right)(1+m)^{-3} \\
& g: m \mapsto 6 m+4 m^{2}+m^{3}
\end{aligned}
$$

intersect at a unique point $m_{*}$ which lies in $(-1,0)$. This proves the following.

Proposition 5.1 The map $\bar{h}$ has a fixed point at $\left(0, m_{*}\right)$ and no other fixed point on the m-axis.

It is easy to check from the graphs of $f, g$ that $f^{\prime}\left(m_{*}\right)>g^{\prime}\left(m_{*}\right)$, and also to verify that

$$
D \bar{h}\left(0, m_{*}\right)=\left(\begin{array}{cc}
1+m & 0 \\
* & f^{\prime}\left(m_{*}\right) / g^{\prime}\left(m_{*}\right)
\end{array}\right) .
$$

This implies the next result.

Proposition 5.2 The map $\bar{h}$ has a hyperbolic saddle fixed point at $\left(0, m_{*}\right)$. The $m$-axis is the unstable manifold, while the stable manifold is a smooth curve $W^{s}$ through $\left(0, m_{*}\right)$ transverse to the $m$-axis.

The interpretation of these results now depends on the sign of $a^{\prime \prime}\left(\tau_{*}\right)$.

If $a^{\prime \prime}\left(\tau_{*}\right)<0$ then $a(\tau)$ has a local maximum at $\tau_{*}$ with $a\left(\tau_{*}\right)=0$, and so from (5) we have $\frac{\partial}{\partial t} v_{c}(\tau, t)>0$ for all $(\tau, t)$ in some neighbourhood of $w_{*}=\left(\tau_{*}, 0\right)$ in $\Pi$ except at $w_{*}$. Therefore $F$ is defined on a neighbourhood of $w_{*}$ in $\Pi$ and is a smooth local homeomorphism onto a neighbourhood of $w_{*}$ in $V^{\prime}$. Hence if $\beta=\chi\left(W^{s}\right)$ then $\gamma=p(\beta) \cap \Pi_{0}^{+}$is a smooth forward invariant curve $\gamma$ for $G$ terminating at the swan point. As is clear from (34) this curve $\gamma$ has cubic tangency with $\Pi_{0}$ at $w_{*}$.

Theorem 5.3 Let $w_{*}=\left(\tau_{*}, 0\right) \in \Pi_{0}$ be a swan point with $a^{\prime \prime}\left(\tau_{*}\right)<0$. There is a smooth arc $\gamma \subset \Pi_{0}^{+}$with an end-point at $w_{*}$, cubically tangent to $\Pi_{0}$ at $w_{*}$ from the side $\tau<\tau_{*}$ and otherwise lying in $\Pi^{+}$. The arc $\gamma$ is forward invariant for $G$ and locally unique in the sense of Theorem 4.1. The fixed point $w_{*}$ is exponentially attracting for $G \mid \gamma$, and is weakly normally hyperbolic (repelling) for $G$. All points in a neighbourhood of $w_{*}$ but not on $\gamma$ lie on stable manifolds of regular points of $\Pi_{0}$ (nondegenerate chatter). See Figure 8 .

Thus with $a^{\prime \prime}\left(\tau_{*}\right)<0$ all initial data close to $w_{*}$ lead to nondegenerate chatter, with the exception of those on the curve $\gamma$ which exhibit a form of highly degenerate chatter.

If on the other hand $a^{\prime \prime}\left(\tau_{*}\right)>0$ then we have $v_{c}(\tau, t)<0$ for sufficiently small $t>0$ and $\tau$ close to $\tau_{*}$, and so the image under $F$ of a neighbourhood of $w_{*}$ in $\Pi_{0}^{+}$will include no points close to $w_{*}$. Therefore the local analysis of low-velocity impacts which is the subject of this paper will not apply.

\section{Unfolding the dynamics at the swan point}

Typically (the precise conditions on the functions $f$ and $g$ in (1) are described in [16]) swan points will occur for only certain isolated values of $c$ or of some other parameter 


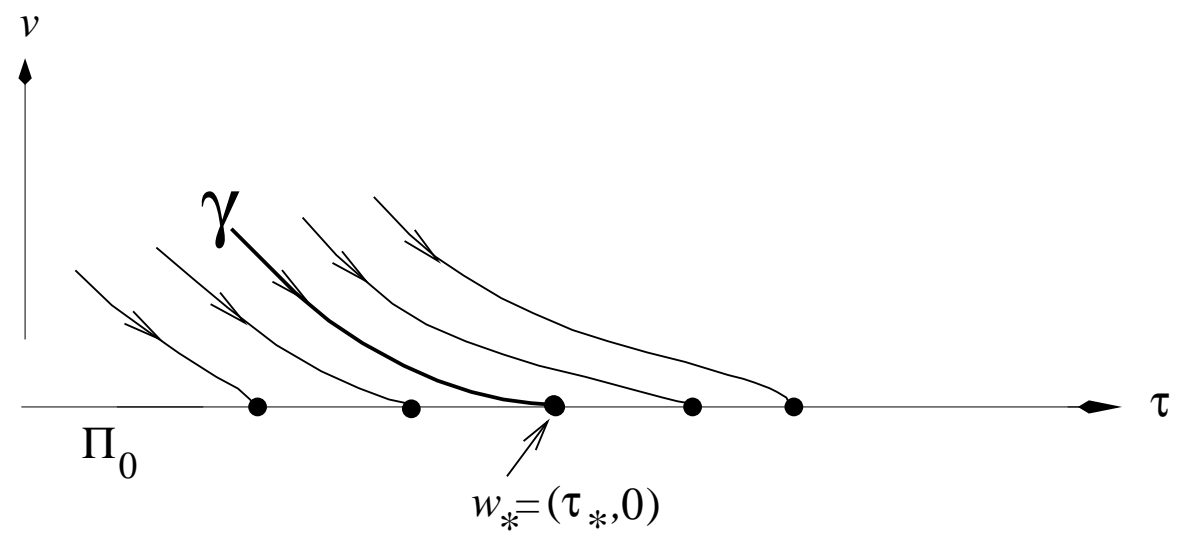

Figure 8. Stable manifolds of chatter points close to a swan point $\left(\tau_{*}, 0\right)$ where $a^{\prime}\left(\tau_{*}\right)=0$ and $a^{\prime \prime}\left(\tau_{*}\right)<0$.

in the system: swan points are codimension-1 phenomena. Therefore it is important to consider the bifurcations in the local dynamics as a generic transition through a swan point takes place.

We shall take the parameter to be $c$ for simplicity, although this is not crucial for the discussion. For clarity we also now re-introduce the $c$ suffix in notation for the maps $p_{c}, \phi_{c}, F_{c}$ and $G_{c}$ and the surface $V_{c}^{\prime}$. We suppose that $a_{c}\left(\tau_{*}\right)=a_{c}^{\prime}\left(\tau_{*}\right)=0$ when $c=c_{0}$ and let

$$
d(c, \tau):=a_{c}^{\prime \prime}(\tau) \frac{\partial a}{\partial c}(c, \tau)
$$

where $a(c, \tau)=a_{c}(\tau)$. As $c$ increases through $c_{0}$ the function $a_{c}(\tau)$ undergoes a Morse transition at which a pair of zeros $\tau_{1}, \tau_{2}$ of $a_{c}(\tau)$ is created or annihilated according to the sign of $d_{0}:=d\left(c_{0}, \tau_{*}\right)$. Note that under the Correspondence Principle the creation of two tangency points at a swan point corresponds to the creation of two cusps at a swallowtail (Figure 2(c)).

Without loss of generality we suppose $d_{0}<0$. As $c$ increases through $c_{0}$ an interval $\left(\tau_{1}, \tau_{2}\right)$ is created on which $a_{c}(\tau)$ reverses its sign, and a new component of $P_{c}$ (a swan configuration) is created out of the point $w_{*}$ as described in [16]. See Figure 9. The creation of the swan is in fact a version of the lips transition (Figure 2(a)) with particular geometry dictated by the context, a characteristic geometry of singular sets noted earlier by Bruce [11].

There are two cases to consider, according to whether $a_{0}^{\prime \prime}\left(\tau_{*}\right):=a_{c_{0}}^{\prime \prime}\left(\tau_{*}\right)>0$ (positive swan point) or $a_{0}^{\prime \prime}\left(\tau_{*}\right)<0$ (negative swan point).

\subsection{Positive swan point: $a_{0}^{\prime \prime}\left(\tau_{*}\right)>0$}

For $c<c_{0}$ there are no low-velocity impacts occurring close to $w_{*}$, and for $c=c_{0}$ although the acceleration vanishes at $\tau_{*}$ there are still no low-velocity impacts. We 

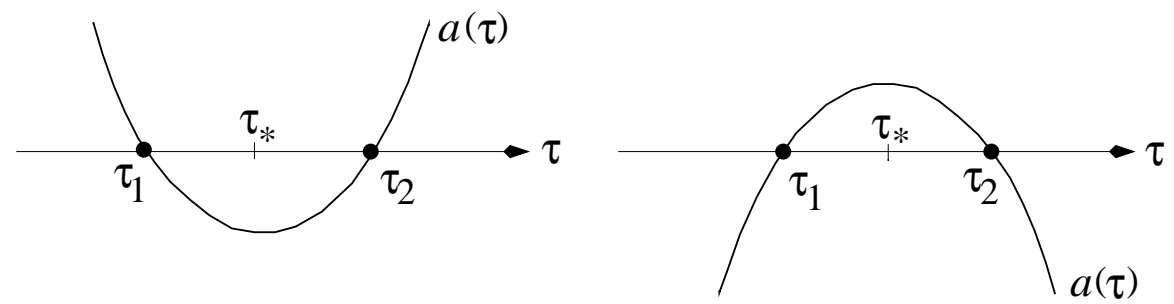

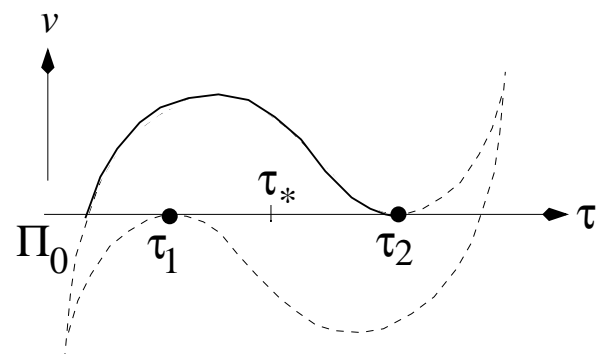

(a)

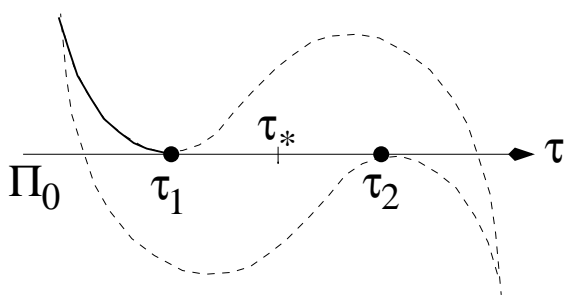

(b)

Figure 9. Graphs of $a(\tau)$ and associated swan configurations in the plane $\Pi$ in two cases: (a) positive, (b) negative.

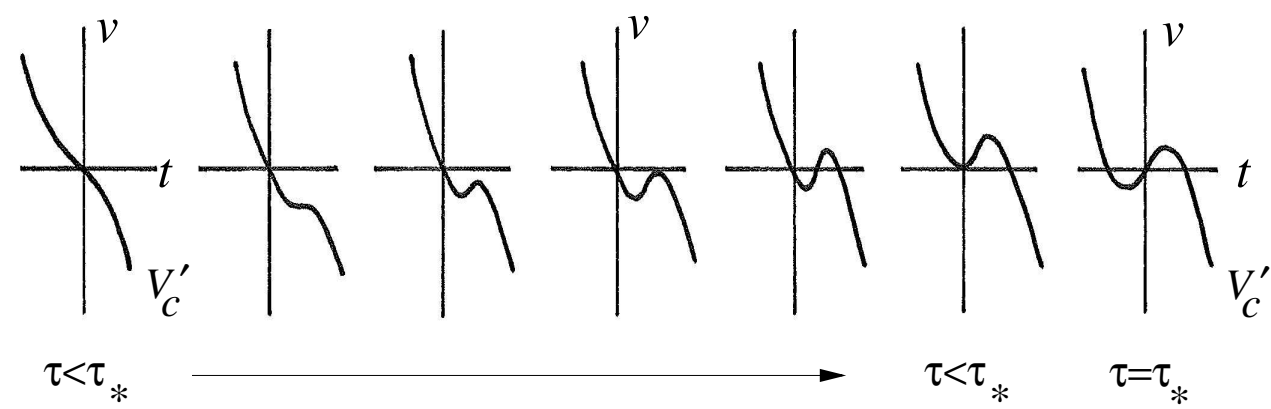

Figure 10. Sections through $V_{c}^{\prime}$ in the $(t, v)$-plane for increasing values of $\tau$ with $\tau \leq \tau_{*}$.

therefore focus on the low-velocity dynamical phenomena that are created when $c>c_{0}$. Once again we now suppose such $c$ fixed sufficiently close to $c_{0}$ and dispense with it as a suffix.

To help picture the dynamics of $G$ (and in particular the geometry of $F$ ) we show in Figure 10 a sequence of sections through $V^{\prime}=V_{c}^{\prime}$ at constant $\tau$ for various choices of $\tau \leq \tau_{*}$. This information can be obtained from the Taylor expansion (11), regarding 


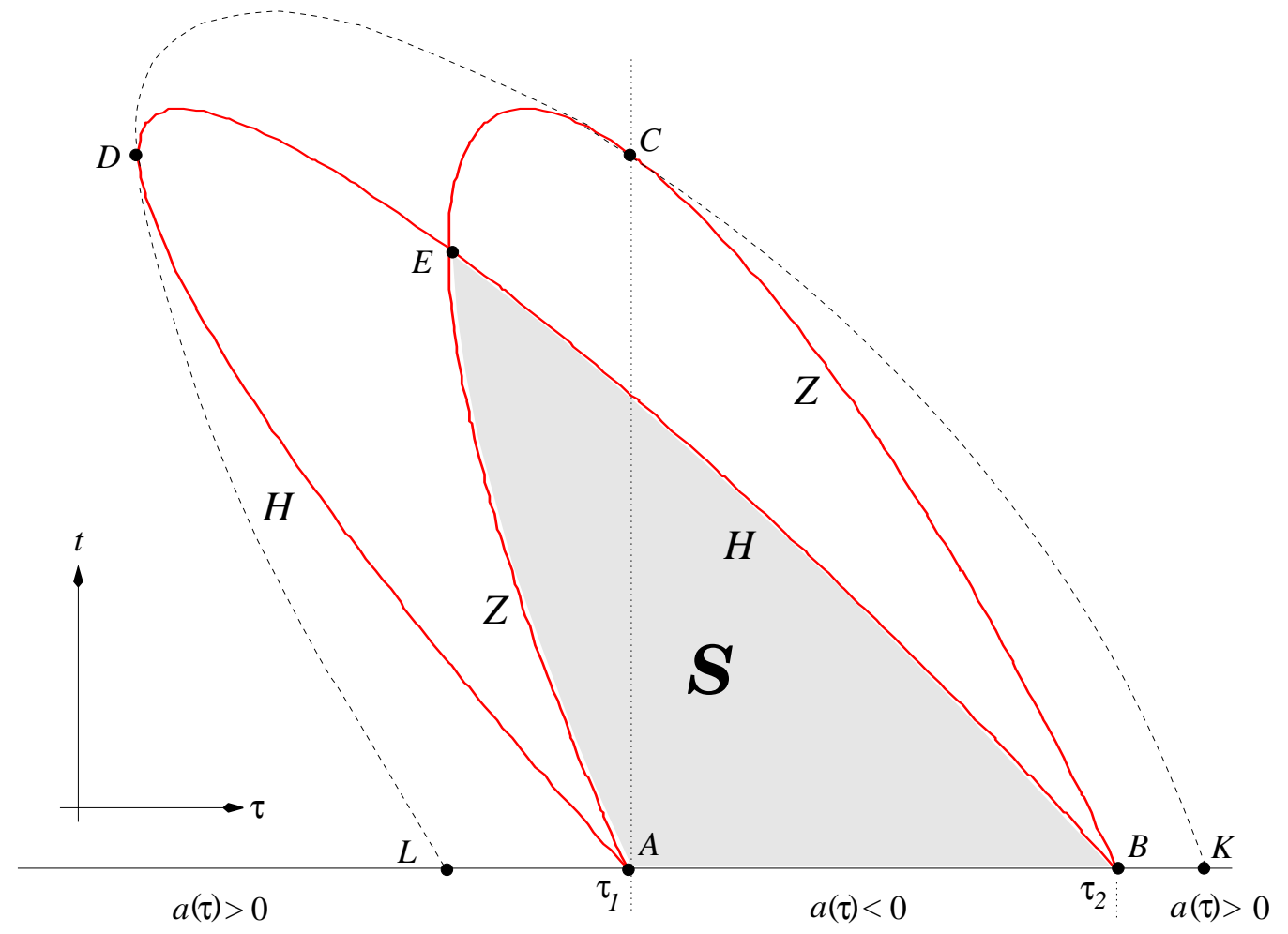

Figure 11. Positive swan configuration: the visible part $\mathcal{S}$ of $V^{\prime}$ is bounded by an arc of the horizon $H$ and an arc of the zero locus $Z$ as well as the interval $A B$ of $\Pi_{0}$. See text for details.

$v_{c}\left(\tau_{*}+\sigma, t\right)$ as a function of $t$ unfolded by the parameter $\sigma$. Analogous sketches for $\tau \geq \tau_{*}$ are obtained by reversing the signs of both $t$ and $v$.

In Figure 11 we show the horizon $H$ and the zero set $Z$ in $(\tau, t)$-coordinates on $V^{\prime}$, thus viewed from the 'vertical' $v$ direction. Here $A=\left(\tau_{1}, 0\right)$ and $B=\left(\tau_{2}, 0\right)$ where $\tau_{1}<\tau_{2}$ are the two zeros of $a(\tau)$ near $\tau_{*}$ for $c>c_{0}$ and close to $c_{0}$. The shaded area $\mathcal{S}$ indicates the connected component of the visible part of $V^{\prime}$ that contains the interval $A B$ (see Section 2.2).

As already seen in Chapter 4 , the dynamics of $G$ close to $A$ and $B$ are as in Figure 5 and Figure 7 respectively. These combine to give the swan dynamics in the way that we now describe.

Figure 12(a) shows the same configuration as Figure 11 but viewed along the $t$ axis, or in other words its image in $\Pi$ under the projection $p$. Points $p(C), p(D), \ldots$ are denoted $C^{\prime}, D^{\prime}, \ldots$; note that $A^{\prime}=A$ and $B^{\prime}=B$. The $\operatorname{arc} B E$ of $H$ is taken by $p$ to a smooth arc $B E^{\prime}$ in $\Pi_{0}^{+}$tangent to $\Pi_{0}$ at $B$ and transverse to $\Pi_{0}$ at $E^{\prime}$ : together with the interval $E^{\prime} B$ of $\Pi_{0}$ it bounds the simply-connected region $\mathcal{S}^{\prime}=p(\mathcal{S}) \subset \Pi_{0}^{+}$.

Figure 12(b) shows the image of the configuration in Figure 11 under the re-set map $\phi$, with points $\phi(C), \phi(D), \ldots$ denoted $C^{\prime \prime}, D^{\prime \prime}, \ldots$ The arc $A E$ of $Z$ is taken by $\phi$ to a smooth arc $A E^{\prime \prime}$ in $\Pi_{0}^{-}$tangent to $\Pi_{0}$ at $A$ and transverse to $\Pi_{0}$ at $E^{\prime \prime}$ (part of the 
apparent outline in view of the Correspondence Principle: see Proposition 2.3); together with the interval $A E^{\prime \prime}$ of $\Pi_{0}$ it bounds the simply-connected region $\mathcal{S}^{\prime \prime}=\phi(\mathcal{S}) \subset \Pi_{0}^{-}$.

To construct the dynamics of $G$ we compose the inverse of $p$ with $\phi$ and then with the restitution $R$. The latter takes the $\operatorname{arc} A E^{\prime \prime}$ in $\Pi_{0}^{-}$to a smooth arc in $\Pi_{0}^{+}$tangent to $\Pi_{0}$ at $A$ and transverse to $\Pi_{0}$ at $E^{\prime \prime}$. Its intersection with $\mathcal{S}^{\prime}$ consists of a smooth arc $\varepsilon^{\prime}$ also tangent to $\Pi_{0}$ at $A$ and with its other end-point $N$ lying on the arc $L B$ of the apparent outline. The arc $\varepsilon^{\prime}$ is the maximal extension (as far as the apparent outline $L B$ ) of the arc $\varepsilon$ in Figure 7 . The $G$-invariant curve $\gamma$ at the degenerate chatter point $B$ is tangent to $\Pi_{0}$ at $B$ and transverse to $\Pi_{0}$ at its other point of intersection. It separates $\mathcal{S}^{\prime}$ into two regions: one foliated by stable manifolds of points of $A B$ (nondegenerate chatter), the other consisting of points that eventually leave $\mathcal{S}^{\prime}$. See Figure 13.

\subsection{Negative swan point: $a_{0}^{\prime \prime}\left(\tau_{*}\right)<0$}

For $c<c_{0}$ we have $a(\tau)<0$ for all $\tau$ in a neighbourhood of $\tau_{*}$ and so Theorem 3.1 applies: every point lies in the stable manifold of some point of $\Pi_{0}$ and nondegenerate chatter takes place locally as in Figure 3(b).

For $c=c_{0}$ Theorem 5.3 applies: there is a smooth stable manifold for $\left(\tau_{*}, 0\right)$ that is cubically tangent to $\Pi_{0}$ and corresponds to highly degenerate chatter, while all other orbits experience nondegenerate chatter becoming increasingly degenerate as $\tau \rightarrow \tau_{*}$ from either side as in Figure 8. We therefore focus on the case $c>c_{0}$.

The analogue of Figure 10 for this case is obtained by inverting the $v$-axis. In Figure 14 we again show the horizon $H$ and the zero set $Z$ as viewed from the $v$ direction, with $A, B$ as before corresponding to the values $\tau_{1}, \tau_{2}$ of $\tau$ where $a(\tau)=0$. Now, however, it is a different region $\mathcal{T}=\mathcal{T}_{1} \cup \mathcal{T}_{2} \cup \mathcal{T}_{3}$ that is visible, bounded by the arc $B C$ of $Z$, the arc $D A$ of $H$, and the (dotted) arc $D C$ which is the shadow of $D A$, that is the set of "next-hit" points starting from $D A$ : more precisely it is the set of points $\left(\tau, v ; t_{1}\right) \in V^{\prime}$ for which there exists $t_{0}>0$ with $\left(\tau, v ; t_{0}\right) \in D A$ but $(\tau, v ; t) \notin V^{\prime}$ for all $t_{0}<t<t_{1}$. The visible part of $V^{\prime}$ is the region lying outside the loop ADCBA (including $A D$ but not including the open arc $D C$ ).

Under the correspondence principle in Figure 14 the points $A, B$ each correspond to cusp points elsewhere on $H$ : in fact $B$ corresponds to $D$ while $A$ corresponds to its counterpart (not shown) with $t<0$. Figure $15\left(\right.$ a) shows the images $A^{\prime}, \ldots, E^{\prime}$ of the points $A, \ldots, E$ of $V^{\prime}$ under projection $p$, while Figure 15(b)shows their images $A^{\prime \prime}, \ldots, E^{\prime \prime}$ under the re-set map $\phi$. The only visible part of $H$ is the arc from $A$ to $D$ : under $p$ this projects to the arc $A D^{\prime}$ in $\Pi_{0}^{+}$while under $\phi$ it is taken to the interval $A^{\prime \prime} D^{\prime \prime}$ on the $\tau$-axis $\Pi_{0}$.

At points of the shadow arc $C D$ apart from $D$ the velocity $\dot{x}_{c}$ is nonzero (in fact it has to be negative) and so $\phi$ takes $C D$ to an $\operatorname{arc} C^{\prime \prime} D^{\prime \prime}$ with $v<0$ except at $D^{\prime \prime}$; moreover $C^{\prime \prime}$ is a cusp point of $P$ by Proposition 2.2. From this we see the nature of the discontinuity of the map $\phi \circ F$ (and hence of $G$ ): cutting $\Pi^{+}$along the arc $A D^{\prime}$ then 

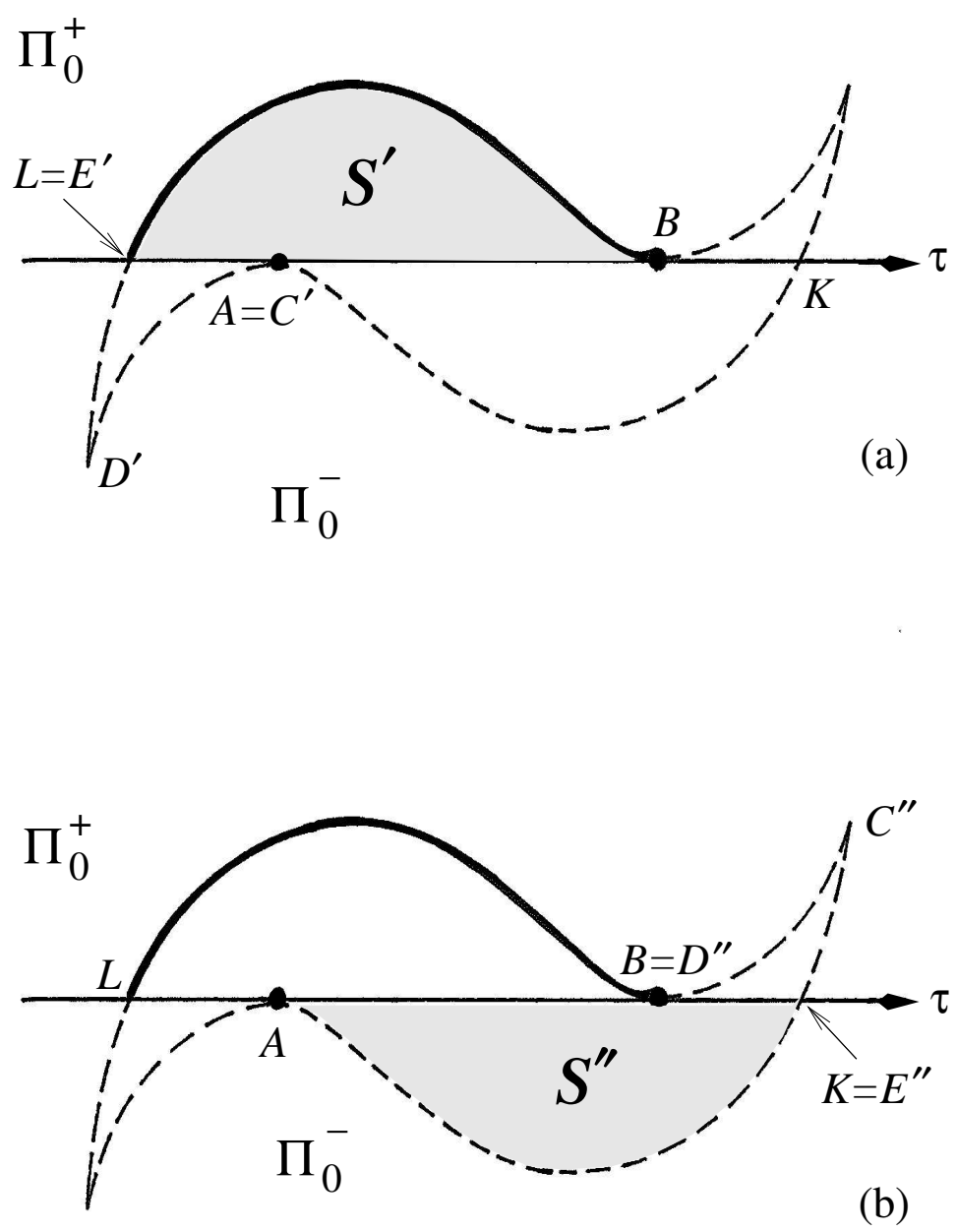

Figure 12. The images under (a) the projection $p$ and (b) the re-set map $\phi$ of various regions of $V^{\prime}$ in Figure 11 for an unfolded positive swan point: see text for details.

mapping one edge (heavy) to the interval $A^{\prime \prime} D^{\prime \prime}$ of $\Pi_{0}$ and the other edge (dotted) to the arc $D^{\prime \prime} C^{\prime \prime}$. Meanwhile the interval $A B$ of Figure $15(\mathrm{a})$ is mapped by $F$ to the arc $C B$ of $Z$ which is then taken by $\phi$ to the $\operatorname{arc} C^{\prime \prime} B$ of Figure $15(\mathrm{~b})$.

These pictures repay contemplation in order to form a clear mental picture of the dynamics of $G$. Figure 16 shows schematically how $\phi \circ F$ takes each segment of the swan region into the next segment; remember, however, that the map $G$ also incorporates the restitution $R$ at each step and takes each of $\Pi_{0}^{+}, \Pi_{0}^{-}$into itself. We now describe the dynamics.

In Section 4.1 we have already obtained local models for the dynamics of $G$ close to the two tangency points $A$ and $B$ (Figure 5 and Figure 7 respectively). As there are 


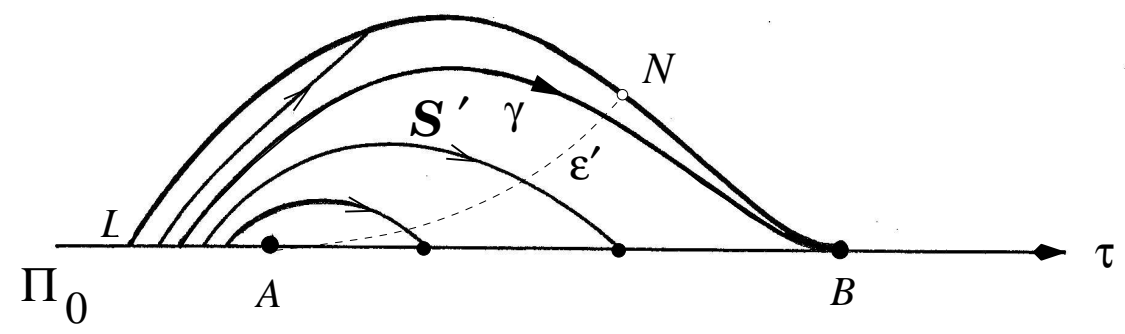

Figure 13. Invariant foliation for dynamics of $G$ in the region $S^{\prime}$. Outside $S^{\prime}$ the unfolding of the swan point gives no local information.

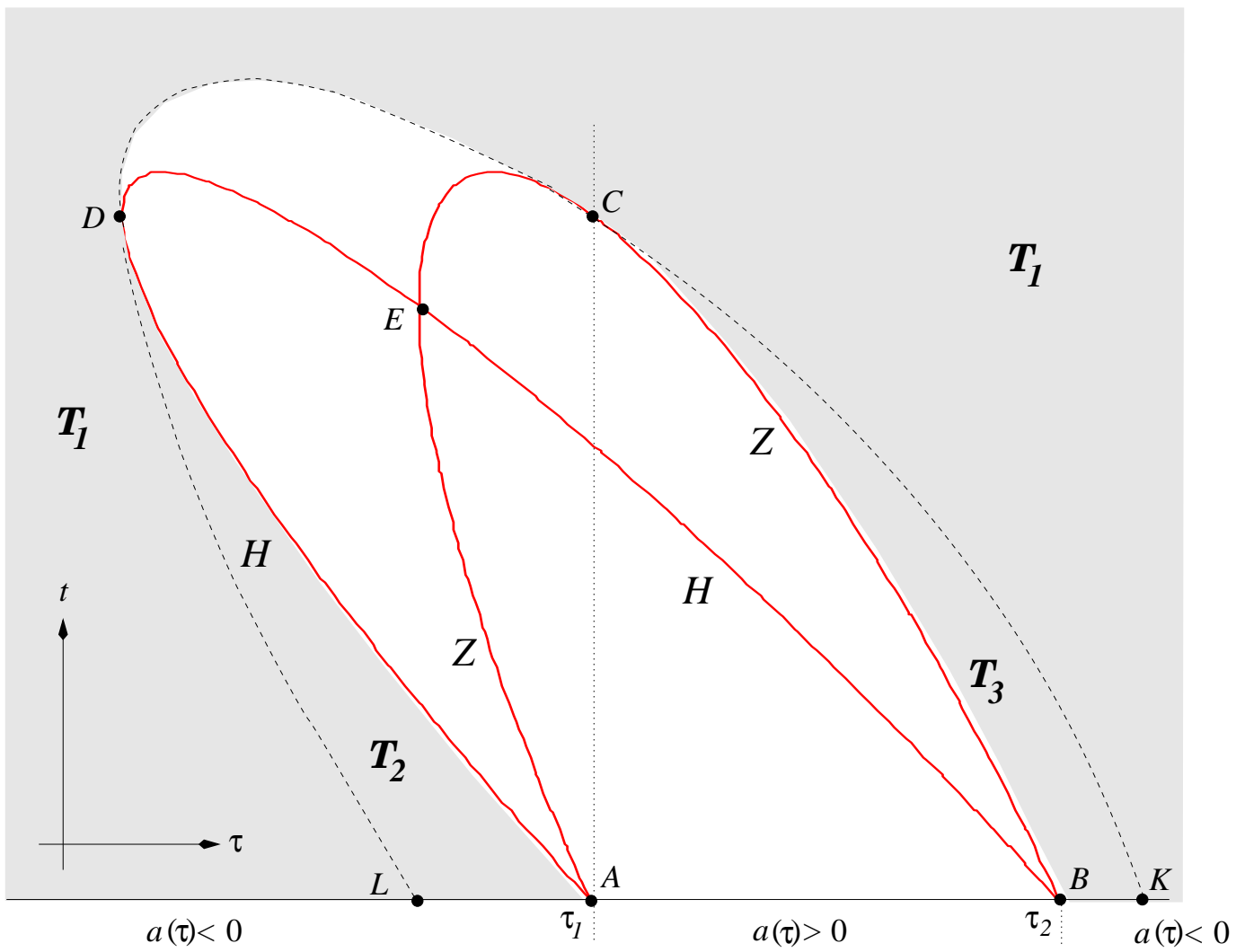

Figure 14. Negative swan configuration on $V^{\prime}$ : the visible part (shaded) of $V^{\prime}$ here is bounded by an $\operatorname{arc}$ of $H$ and an $\operatorname{arc}$ of $Z$ together with an $\operatorname{arc} D C$ of the shadow of $H$.

no discontinuities of $G$ close to $B$, the neighbourhood of $B$ that is foliated by stable manifolds $W_{\tau}^{s}$ of points $(\tau, 0)$ with $\tau>\tau_{2}$ (by Theorem 4.6) must extend at least as far as the stable manifold $W_{\tau_{3}}^{s}=W^{s}(X)$ that contains the point $A$, where $X=\left(\tau_{3}, 0\right)$ with 


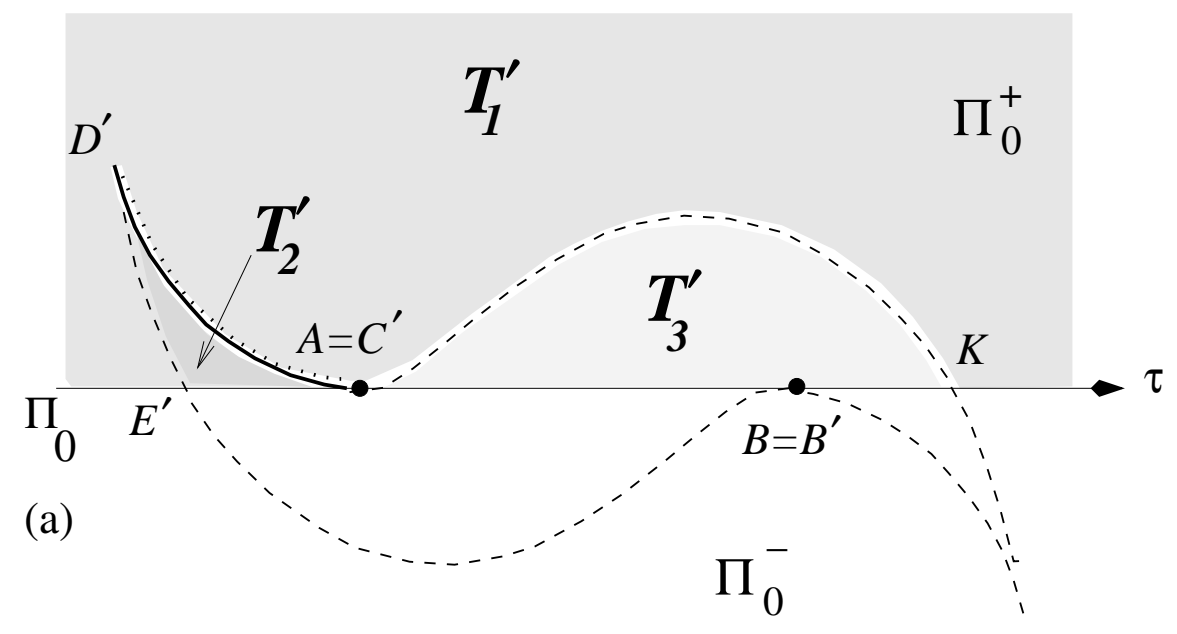

(b)

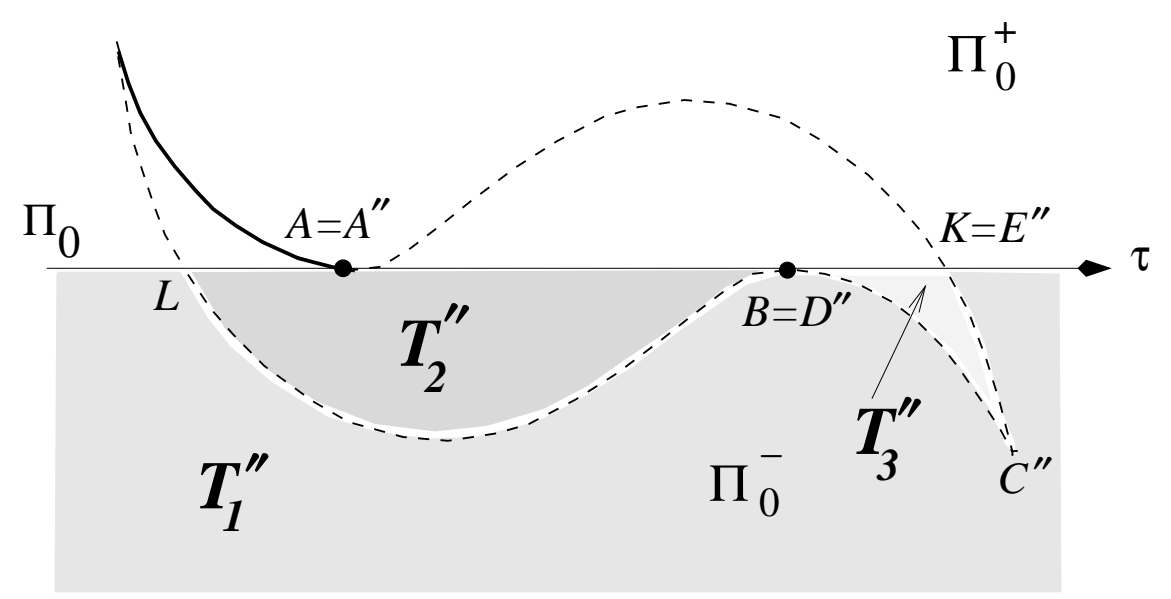

Figure 15. The images under (a) the projection $p$ and (b) the re-set map $\phi$ of various regions of $V^{\prime}$ for an unfolded negative swan point: see text for details.

$\tau_{3}>\tau_{2}$. See Figure 17

Proposition 6.1 The stable manifold $W^{s}(X)$ is tangent to $\Pi_{0}$ at $A$.

Proof. We argue that $F\left(W^{s}(X)\right) \subset V^{\prime}$ is tangent to $Z$ at $C$, from which the result follows by applying $p$.

Since the map $\phi$ has a cusp singularity at $C$ by Proposition 2.2, if $F\left(W^{s}(X)\right)$ were not tangent to $Z$ at $C$ then $\phi \circ F\left(W^{s}(X)\right)$ would be aligned with the direction of the cusp at $C^{\prime \prime}$ in Figure 15. In that case $R \circ \phi \circ F\left(W^{s}(X)\right)=G\left(W^{s}(X)\right)=W^{s}(X)$ would be tangent to the arc $\varepsilon^{\prime \prime}=R\left(D^{\prime \prime} C^{\prime \prime}\right)$ at its endpoint $R\left(C^{\prime \prime}\right)$, and this is inconsistent with the geometry of chatter close to the point $B$ where $a(\tau)=0$ and $a^{\prime}(\tau)<0$ as in Figure 7: stable manifolds of points of $\Pi_{0}$ to the right of $B$ are transverse to $\varepsilon^{\prime \prime}$.

Let $\lambda$ denote the arc of $W^{s}(X)$ from $A$ to $Q=W^{s}(X) \cap \varepsilon^{\prime \prime}$. The inverse image 


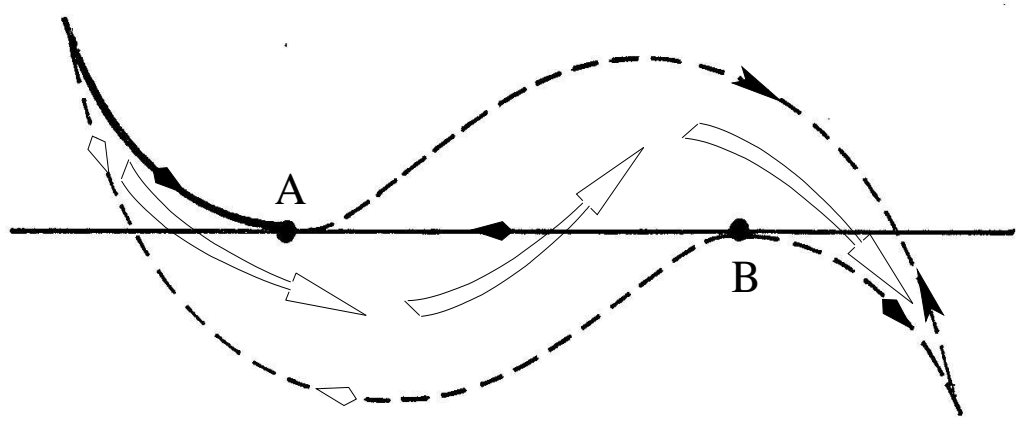

Figure 16. Schematic representation of the action of $\phi \circ F$ within the swan region. Matching arrows indicate arcs and their images. The third step is discontinuous along $A B$.

$G^{-1}(\lambda)$ is a closed loop around the discontinuity $\operatorname{arc} \delta=A D^{\prime}$ and with both ends at $A$. The inverse images of $\delta$ under iterates of $G$ form a sequence of arcs tangent to $\Pi_{0}$ at the degenerate chatter point $A$ and accumulating on its invariant arc $\gamma$, and therefore the inverse images of $\lambda$ form a sequence of loops also accumulating on $\gamma$. Stable manifolds $W^{s}(Y)$ of points $Y$ to the right of $X$ on $\Pi_{0}$ (i.e. with $\tau>\tau_{3}$ ) and close to $X$ oscillate more and more wildly under backwards iteration of $G$ as they attempt to adhere to the loops of $G^{-n}(\lambda)$. Again see Figure 17 .

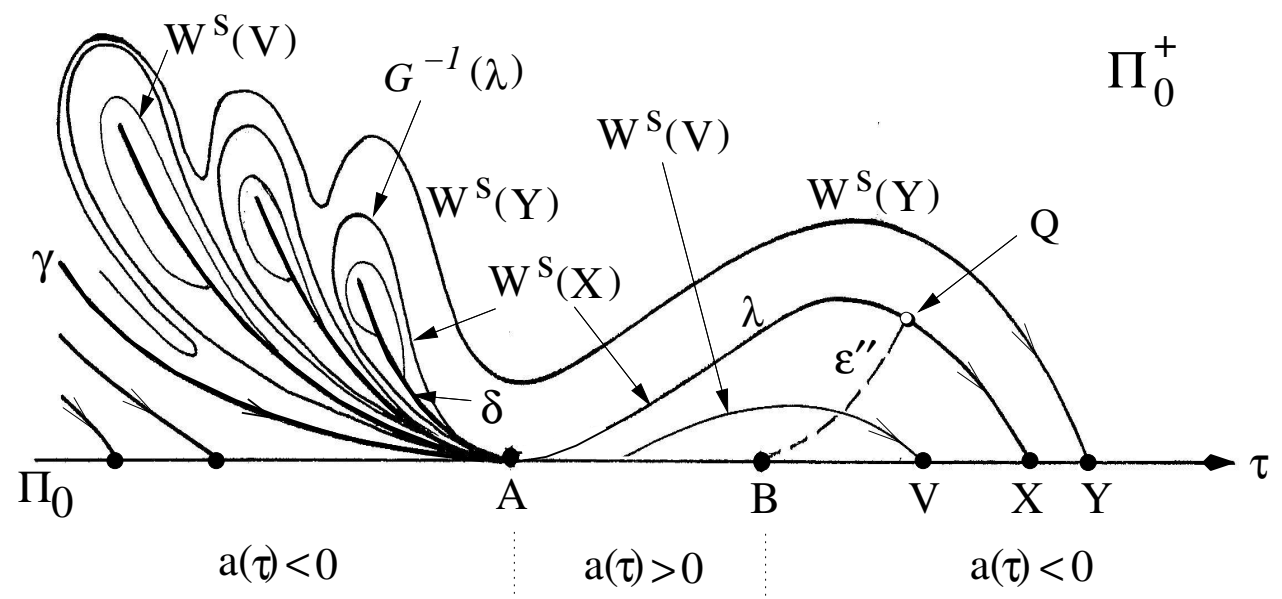

Figure 17. Dynamics of $G$ created at unfolding of a negative swan point: see text for details. 


\section{Global dynamics}

The dynamical phenomena that become apparent in the unfolding of a negative swan point give important clues about the global geometry for dynamics of an impact oscillator. In particular we see that the particular geometry of the smooth map $p \circ \phi^{-1}$ close to a tangency point $w_{*}=\left(\tau_{*}, 0\right) \in \Pi_{0}$ (so that $z=\phi^{-1}\left(w_{*}\right)$ is a cusp point of $H$ ) plays a key role. At a tangency point $w_{*}$ where $a^{\prime}\left(\tau_{*}\right)>0$ the nearby stable manifolds $W_{\tau}^{s}$ with $\tau>\tau_{*}$ have infinitely many loops accumulating on the forward invariant curve $\gamma$ that is the stable manifold of $w_{*}$ Therefore we see that the global behaviour of the stable manifolds of degenerate chatter points, and in particular their intersections with unstable manifolds of fixed or periodic points elsewhere in the phase space $\Pi_{0}^{+}$, are crucial in organising the global effects of the complicated dynamics unleashed at a (negative) swan bifurcation. The swan configuration created at a negative swan point will persist until variation in the parameter $c$ causes it to impinge on some other branch of the apparent outline $P$. Careful study of the generic ways in which this can happen will provide important tools for understanding the complicated global structure of discontinuous planar discrete dynamical systems modelling 1-degree of freedom impact oscillators.

\section{Acknowledgments}

I am most grateful to the Centre de Recerca Matemàtica, Barcelona, for hospitality during the preparation of the main part of this paper as part of the Research Programme on Complex Non-Smooth Dynamical Systems organised there by the Bristol Centre for Applied Nonlinear Mathematics in Spring 2007. I thank colleagues at BCANM for hospitality and continuing interest in and support for this research.

\section{References}

[1] Alzate R, di Bernardo M, Montanaro U and Santini S 2007 Experimental and numerical verification of bifurcations and chaos in cam-follower impacting systems J Nonlinear Dynamics 50 409-429 doi 101007/s11071-006-9188-8

[2] Andronov A A, Vitt A A and Khaikin S E 1959 Theory of Oscillations, Moscow; Eng. transl. Pergamon Press 1966

[3] Arnol'd V I 1986 Catastrophe Theory, 2nd ed (Berlin: Springer)

[4] Arnol'd V I, Gusein-Zade S M and Varchenko A N 1985 Singularities of Differentiable Maps, Vol I, (Boston, Mass.: Birkhäuser)

[5] Babitsky V I and Birkett N 1988 Theory of Vibro-Impact Systems and Applications, (Berlin Heidelberg: Springer-Verlag)

[6] di Bernardo M, Budd C J, Champneys A R, Kowalczyk P, Nordmark A B, Tost G O and Piiroinen P T 2008 Bifurcations in nonsmooth dynamical systems SIAM Review 50 629-701

[7] di Bernardo M, Budd C J, Champneys A R and Kowalczyk P 2008 Piecewise-Smooth Dynamical Systems: Theory and Applications (London: Springer-Verlag)

[8] Brogliato B 1999 Nonsmooth Mechanics London: Springer-Verlag) 
[9] Bruce J W 1984 Motion pictures: an application of singularity theory J London Math Soc 30 $160-170$

[10] Bruce J W 1984 Seeing - the mathematical viewpoint Math Intelligencer 6 18-25

[11] Bruce J W 1989 Geometry of singular sets Math Proc Cambridge Philos Soc 106 495-509

[12] Bruce J W and Giblin P J 1984 Curves and Singularities (Cambridge: Cambridge University Press)

[13] Bruce J W and Giblin P J 1985 Outlines and their duals Proc London Math Soc 50 552-570

[14] Budd C J and Dux F J 1994 Chattering and related behaviour in impact oscillators Phil Trans $R$ Soc London A 347 365-389

[15] Budd C J, Dux F J and Cliffe A 1995 The effect of frequency and clearance variations on singledegree-of-freedom impact oscillators $J$ Sound and Vibration 184 475-502

[16] Chillingworth D R J 2002 Discontinuity geometry for an impact oscillator Dynamical Systems 17 $389-420$

[17] Chin W, Ott E, Nusse H E and Grebogi C 1994 Grazing bifurcation in impact oscillators Phys Rev E 50 4427-4444

[18] Dankowicz H and Jerrelind J 2005 Control of near-grazing dynamics in impact oscillators Proc Roy Soc London A 461:2063 3365-3380

[19] Demeio L and Lenci S 2006 Asymptotic analysis of chattering oscillations for an impacting inverted pendulum Quarterly J Mech Appl Math 59 419-434

[20] Fidlin A 2006 Nonlinear Oscillations in Mechanical Engineering (Berlin Heidelberg: SpringerVerlag)

[21] Foale S and Bishop S R 1992 Dynamical complexities of forced impacting systems Phil Trans R Soc London A 338 547-556

[22] Golubitsky M and Guillemin V 1973 Stable Mappings and their Singularities (New York: SpringerVerlag)

[23] Guckenheimer J and Holmes P 1983 Nonlinear Oscillations Dynamical Systems and Bifurcations of Vector Fields (New York: Springer) (revised ed. 2002)

[24] Hayashi C 1985 Nonlinear Oscillations in Physical Systems (Princeton: Princeton University Press)

[25] Hindmarsh M and Jeffries D 1984 On the motion of the offset impact oscillator J Physics A: Math Gen 17 1791-1803

[26] Hirsch M W, Pugh C C and Shub M 1977 Invariant Manifolds Lect Notes in Math 583 SpringerVerlag

[27] Hasselblatt B and Pesin Ya 2005 Partially hyperbolic dynamical systems in Handbook of Dynamical Systems IB Elsevier

[28] Lamba H 1995 Chaotic regular and unbounded behaviour in the elastic impact oscillator Physica D 82 117-135

[29] Ivanov A P 1994 Impact oscillations: linear theory of stability and bifurcations $J$ Sound and Vibration 178 361-378

[30] Kleczka M, Kreuzer E and Schiehlen W 1992 Local and global stability of a piecewise linear oscillator Phil Trans R Soc London A 338 533-546

[31] Mason J F, Homer M E and Wilson R E 2007 Mathematical models of gear rattle in Roots blower vacuum pumps $J$ Sound and Vibration 308 431-440

[32] Molenaar J, de Weger J G and van de Water W 2001 Mappings of grazing-impact oscillators Nonlinearity 14 301-321

[33] Nordmark A B 1991 Non-periodic motion caused by grazing incidence in an impact oscillator $J$ Sound and Vibration 145 279-297

[34] Nordmark A B 1997 Universal limit mapping in grazing bifurcations Phys Rev E 55 266-270

[35] Pavlovskaia E and Wiercigroch M 2003 Modelling of vibro-impact system driven by beat frequency Int J Mech Sci 45 623-641

[36] Peterka F and Vacík J 1992 Transition to chaotic motion in mechanical systems with impacts $J$ Sound and Vibration 154 95-115 
[37] Schmidt G and Tondl A 1987 Non-Linear Vibrations (Cambridge: Cambridge University Press)

[38] Shaw S W and Holmes P J 1983 A periodically forced piecewise linear oscillator $J$ Sound and Vibration 90 129-155

[39] Sotomayor J and Teixeira M A 1988 Vector fields near the boundary of a 3-manifold pp 169-195 in Lecture Notes in Mathematics v. 1331 Springer

[40] Stewart D 2000 Rigid-body dynamics with friction and impact SIAM Review 42 3-39

[41] Stone E and Askari A 2002 Nonlinear models of chatter in drilling processes Dynamical Systems $1765-85$

[42] Szalai R and Osinga H M 2008 Unstable manifolds of a limit cycle near grazing Nonlinearity 21 $273-284$

[43] Takens F 1971 Partially hyperbolic fixed points Topology $10133-147$

[44] Thompson J M T and Ghaffari R 1982 Chaos after period doubling bifurcations in the resonance of an impact oscillator Phys Lett 91A 5-8

[45] Valente A X C N, McClamroch N H and Mesić I 2003 Hybrid dynamics of two coupled oscillators that can impact a fixed stop Int J Non-Linear Mech 38 677-689

[46] Virgin L N and Begley C J 1999 Grazing bifurcations and basins of attraction in an impact-friction oscillator Physica D $13043-57$

[47] Wagg D J and Bishop S R 2001 Chatter sticking and chaotic impacting motion in a two-degree of freedom impact oscillator Int $J$ Bifurcation and Chaos 11 57-71

[48] Wagg D J and Bishop S R 2004 Dynamics of a two degree of freedom vibro-impact system with multiple motion limiting constraints Int J Bifurcation and Chaos 14 119-140

[49] Whiston G W 1987 The vibro-impact response of a harmonically excited and preloaded onedimensional linear oscillator $J$ Sound and Vibration 115 303-319

[50] Whiston G W 1987 Global dynamics of a vibro-impacting linear oscillator J Sound and Vibration $118395-429$

[51] Whiston G W Singularities in vibro-impact dynamics 1992 J Sound and Vibration 152 427-460

[52] Whitney H On singularities of mappings of Euclidean spaces I 1955 Mappings of the plane into the plane Ann Math 62 374-410

[53] Zhao X and Dankowicz H 2006 Unfolding degenerate grazing dynamics in impact actuators Nonlinearity 19 (2006) 399-418 